\title{
Ratios of Parameters: Some Econometric Examples
}

\author{
Jenny Lye and Joe Hirschberg*
}

\begin{abstract}
Ratios of parameter estimates are often used in econometric applications. However, constructing confidence intervals for these ratios can cause difficulties since the ratio of asymptotically normally distributed random variables are Cauchy distributed thus they have no finite moments.

This paper presents a method for the estimation of confidence intervals based on the Fieller approach that has been shown to be preferable to the usual Delta method. Using example applications in Stata and R, we demonstrate that a few extra steps in the examination of the estimate of the ratio can provide a confidence interval with superior coverage.
\end{abstract}

Key words: turning points, quadratic specification, interaction terms, long-run elasticities, willingness-to-pay, NAIRU, 2SLS \& ILS, Stata

JEL: C12, A22, C51

*Department of Economics, University of Melbourne, Victoria,Australia, 3010. Corresponding author: Lye, email jnlye@unimelb.edu.au

This is the author manuscript accepted for publication and has undergone full peer review but has not been through the copyediting, typesetting, pagination and proofreading process, which may lead to differences between this version and the Version of Record. Please cite this article as doi: 10.1111/1467-8462.12300 


\section{Introduction}

Many econometric applications draw inferences from a parameter of interest that is defined as the ratio of regression coefficients. However, the properties of the ratio of estimates can be problematic. This paper examines eight examples of econometric models where inferences for ratios are used. These examples include; the interpretation of a dummy variable in terms of changes in a continuous regressor, the location of a turning point in a quadratic specification where the marginal impact of a regressor changes sign, the interpretation of the marginal effect of one regressor when interacted with another, the estimation of the long-run effect in a dynamic model, the determination of the "Taylor Rule" for dynamic macro models, the determination of the nonaccelerating inflation rate of unemployment (NAIRU), the willingness to pay, and the structural parameter in an exactly identified system of equations as estimated by the two-stage least squares method. We present analysis of applications of each of these cases along with the corresponding Stata code to obtain these results.

In this paper, we emphasise the use of confidence intervals (CIs) instead of the use of pvalues. Because confidence intervals are expressed in the units of the parameter of interest instead of as a probabilistic abstraction, we concentrate on the nature of these intervals. The traditional approach for constructing CIs is based on the Delta method where a first order Taylor-series expansion is used to approximate a linear relationship between the estimated parameters and the ratio. This method is the standard approach to the estimation of CIs and tests of hypothesis for nonlinear functions of regression parameter estimates and is available in most econometric/statistics software.

An alternative method for the construction of CIs for ratios is Fieller's 1954 proposal. This approach has been shown to be superior to the application of the Delta method in several applications (e.g. Hirschberg and Lye 2010c). This superiority has been found in the coverage of the resulting tests where the estimated $100(1-\alpha) \%$ interval from the Fieller method more closely coincides with the theoretical interval than the alternate Delta interval. ${ }^{1}$ In addition, Fieller intervals are not forced to be symmetric as are the Delta intervals. However, in some cases, the Fieller approach may not result in a finite CI for some values of $\alpha$. In such cases the resulting CI may also be the complement of a finite interval or the whole real line. The advantage to the graphical approach presented here is that it can provide an indication of when this may be the case and can provide partial information that can be used to construct open ended intervals. This is shown in some of the examples below. 
Another attraction to the Fieller method is the ease of computation. We demonstrate that this method can be computed using a direct computational method similar to the Delta and does not require the use of simulations and sampling strategies as would be needed when employing a Bootstrap or Bayesian method.

In the next section, we outline the aspects of the theory involved for the Fieller as it can be applied to the ratios of regression coefficients and discuss the syntax of Stata commands we employ to construct the CIs. In Section 3 we provide eight examples of econometric applications where the result of interest is a ratio of parameters or the ratio of linear combinations of parameters as estimated from standard regressions, probit models, systems of equations, and non-linear models.

\section{Confidence Intervals for a ratio of regression coefficients}

\subsection{Ratios of Regression Parameters}

Consider the general linear model,

$$
\mathbf{Y}=\mathbf{X}(\mathbf{B})+\mathbf{e}
$$

where $\mathbf{Y}$ is an $(T \times 1)$ vector of observations on the dependent variable, $\mathbf{X}(\mathbf{B})$ is a $(T \times 1)$ function of an $(T \times k)$ matrix $\mathbf{X}$ of regressors and a $(k \times 1)$ vector $\mathbf{B}$ of unknown parameters, and $\mathbf{e}$ is an $(T \times 1)$ vector of disturbances with mean 0 and $(T \times T)$ covariance matrix $\Omega$. Suppose interest lies in the ratio of regression coefficients defined by,

$$
\theta=\frac{\rho}{\phi}=\frac{H^{\prime} \mathbf{B}+h}{L^{\prime} \mathbf{B}+l}
$$

where $H$ and $L$ are $(k \times 1)$ vectors of known constants thus $H \mathbf{B}$ and $L^{\prime} \mathbf{B}$ are linear combinations of the parameters and $k$ and $l$ are constants. The usual estimate of the ratio $\theta$ in (2.1.2) is,

$$
\hat{\theta}=\frac{\hat{\rho}}{\hat{\phi}}=\frac{H \hat{\mathbf{B}}+h}{L^{\prime} \hat{\mathbf{B}}+l}
$$

where $\hat{\mathbf{B}}$ is the $(k \times 1)$ vector of the estimates of $\mathbf{B}$ in (2.1.1).

The models we consider here assume the estimates of $\mathbf{B}$ are normally distributed with a covariance matrix that we can approximate. In general, regression, maximum likelihood estimation and non-linear least squares all result in estimates of the parameters under these assumptions. ${ }^{2}$ 


\subsection{The Delta Method for CIs}

The estimated variance of $\hat{\theta}$ based on the Delta method is defined for the ratio of the parameter estimates. We assume that the parameter estimates are bivariate normally distributed: ${ }^{3}$

$$
\left[\begin{array}{l}
\hat{\rho} \\
\hat{\phi}
\end{array}\right] \sim N\left\{\begin{array}{l}
\rho \\
\phi
\end{array}\left[\begin{array}{ll}
\sigma_{1}^{2} & \sigma_{12} \\
\sigma_{12} & \sigma_{2}^{2}
\end{array}\right]\right\}
$$

and $\hat{\theta}=\hat{\rho} / \hat{\phi}$.

The estimate of the variance of the ratio of normally distributed random variables based on the Delta method is defined as: (see example 5.5.27 in Casella and Berger 2002)

$$
\begin{aligned}
\operatorname{var}(\hat{\theta}) & \approx(\hat{\rho} / \hat{\phi})^{2}\left[\left(\sigma_{1}^{2} / \hat{\rho}^{2}\right)+\left(\sigma_{2}^{2} / \hat{\phi}^{2}\right)-2\left(\sigma_{12} / \hat{\phi} \hat{\rho}\right)\right] \\
& \approx \hat{\phi}^{-2}\left[\sigma_{1}^{2}+\hat{\theta}^{2} \sigma_{2}^{2}-2 \hat{\theta} \sigma_{12}\right]
\end{aligned}
$$

When using estimates of the variance covariance matrix, the Delta method $100(1-\alpha) \%$ CI for $\hat{\theta}$ is:

$$
\mathrm{CI}(\hat{\theta})_{\text {Delta }}=\hat{\theta} \pm t_{\alpha / 2}\left(\hat{\phi}^{-1} \sqrt{\hat{\sigma}_{1}^{2}+\hat{\theta}^{2} \hat{\sigma}_{2}^{2}-2 \hat{\theta} \hat{\sigma}_{12}}\right)
$$

where $t_{\alpha / 2}$ is the value from the $t$ distribution with $T-k$ degrees of freedom.

\subsection{The Fieller Method for CIs}

The Fieller method (Fieller 1932, 1954, Rao 1973, pp 241-242) provides a general procedure for constructing confidence limits for statistics defined as ratios. A $100(1-\alpha) \%$ Fieller CI for $\theta$ can be found by inverting the $t$-test associated with the null hypothesis based on a linear combination of the parameters defined as: $H_{0}: \rho-\phi \theta_{j}=0$ for different possible values of $\theta$ as designated by $\theta_{j}$. Based on our assumption that the parameter estimates are normally distributed we find that the linear combination of the parameter estimates $(\hat{\rho}, \hat{\phi})$ for any $\theta_{j}$ is also normally distributed:

$$
\left(\hat{\rho}-\hat{\phi} \theta_{j}\right) \sim N\left(0,\left(\rho^{2} \sigma_{1}^{2}-2 \rho \phi \sigma_{12}+\phi^{2} \sigma_{2}^{2}\right)\right)
$$

Or we can rewrite this expression in terms of a standard normal distribution as:

$$
\frac{\left(\hat{\rho}-\hat{\phi} \theta_{j}\right)}{\sqrt{\left(\rho^{2} \sigma_{1}^{2}-2 \rho \phi \sigma_{12}+\phi^{2} \sigma_{2}^{2}\right)}} \sim N(0,1)
$$


By squaring both sides and using the estimated variance of the linear combination we can specify (2.3.2) in terms of the square of a $t$-distribution for a $100(1-\alpha) \% \mathrm{CI}$ as:

$$
\frac{\left(\hat{\rho}-\hat{\phi} \theta_{j}\right)^{2}}{\left(\hat{\rho}^{2} \hat{\sigma}_{1}^{2}-2 \hat{\rho} \hat{\phi} \hat{\sigma}_{12}+\hat{\phi}^{2} \hat{\sigma}_{2}^{2}\right)}=t_{\alpha / 2}^{2},
$$

The values of $\theta$ that would satisfy (2.3.3) define the bounds of the confidence interval - in this way we have "inverted" the test statistic to define the confidence interval.

By moving the denominator in (2.3.3) to the right hand side and combining terms this expression can be written as a quadratic equation in the limiting values of $\theta$ designated as $\tilde{\theta}$ that solve the quadratic equation: $a \tilde{\theta}^{2}+b \tilde{\theta}+c=0$, where $a=\hat{\phi}^{2}-t_{\alpha / 2}^{2} \hat{\sigma}_{2}^{2}, b=2\left[t_{\alpha / 2}^{2} \hat{\sigma}_{12}-\hat{\phi} \hat{\rho}\right]$ and $c=\hat{\rho}^{2}-t_{\alpha}^{2} \hat{\sigma}_{1}^{2}$. The two roots of the quadratic equation,

$$
\mathrm{CI}(\hat{\theta})_{\text {Fieller }}=\left\{\tilde{\theta}_{\text {lower }}, \tilde{\theta}_{\text {upper }}\right\}=\frac{-b}{2 a} \pm \frac{\sqrt{b^{2}-4 a c}}{2 a},
$$

define the Fieller $100(1-\alpha) \%$ CI for $\hat{\theta}$. In general, $\frac{-b}{2 a} \neq \hat{\theta}$ thus unlike the Delta CI, the Fieller CI will not necessarily be symmetric about the estimate of the ratio. Two finite bounds can be derived if the hypothesis $\mathrm{H}_{0}: \phi \neq 0$ can be rejected when $\alpha$ is the level of significance (Buonaccorsi 1979). In addition to the finite interval case, the Fieller CI may be the complement of a finite interval when $\left(b^{2}-4 a c>0, a<0\right)$ or of the whole real line when $b^{2}-4 a c<0, a<0$. These conditions are discussed in Scheffé (1970) and Zerbe (1982).

Hirschberg and Lye (2010a) present a line plot representation of the 100(1- $\alpha) \%$ Fieller CI for $\theta$. The linear combination $\left(\hat{\rho}-\hat{\phi} \theta_{j}\right)$ for varying values of $\theta_{j}$ is plotted along with its corresponding $100(1-\alpha) \%$ confidence bounds defined as:

$$
\left(\hat{\rho}-\hat{\phi} \theta_{j}\right) \pm t_{\alpha / 2}^{2} \sqrt{\left(\hat{\rho}^{2} \hat{\sigma}_{1}^{2}-2 \hat{\rho} \hat{\phi} \hat{\sigma}_{12}+\hat{\phi}^{2} \hat{\sigma}_{2}^{2}\right)}
$$

The estimate $\hat{\theta}$ is found by determining the values of $\theta_{j}$ where $\left(\hat{\rho}-\hat{\phi} \theta_{j}\right)=0$. Similarly, the bounds of the $100(1-\alpha) \% \mathrm{CI}(\hat{\theta})_{\text {Fieller }}$ can be found by determining for what values of $\theta_{j}$ the two functions defined in (2.3.5) are equal to zero. 


\subsection{Stata Commands 4}

As an example, suppose the regression model is,

$$
y=\beta_{1}+\beta_{2} x_{2}+\beta_{3} x_{3}+\varepsilon
$$

and the ratio of interest is defined as,

$$
\theta=\frac{\rho}{\phi}
$$

where $\rho=\beta_{1}+2 \beta_{2}+h, h$ is a constant in this case $=0.5$ and $\phi=1-\beta_{3}$.

We first estimate the model using an appropriate estimation technique and standard errors. For example, suppose we estimate the model using OLS and store the results for later use with the name "reg".

regress y $\times 2 \times 3$

estimates store reg

To find the limits of the delta 95\% CI we use the Stata command nlcom. Within this command we need to define the numerator $\hat{\rho}$, the denominator $\hat{\phi}$ and the ratio $\hat{\theta}=\hat{\rho} / \hat{\phi}$, where in this case we define the formula for each in Stata by: $\left.\hat{\rho}=\left(\_b\left[\_c o n s\right]+2^{*} \_b[x 2]+.5\right)\right)$ and $\hat{\phi}=\left(1-\_b[\times 3]\right) .{ }^{5}$ The Stata command ncolm can be used to define $\hat{\rho}, \hat{\phi}$ and $\hat{\theta}$ with the command syntax:

ncolm (rho: (_b[_cons] +2*_b[x2] + .5) ) (phi: $(1$-_b[x3])) ///

(theta:_b[_cons] $\left.\left.+2^{*} \_b[x 2]+.5\right) /\left(1-\_b[x 3]\right)\right)$, post

The sequence of these definitions is such that we can retrieve the variance and covariance matrix of the numerator and denominator as the first two rows and columns of the covariance matrix stored when using ncolm with the post option (here defined as the matrix V). To find the limits of the Fieller 95\% CI by solving the two roots of the quadratic defined in (2.3.4) we first define $\hat{\rho}$ rho and $\hat{\phi}$ phi and their variances $v 11, \mathrm{v} 22$ and their covariance $v 12$ by the following:

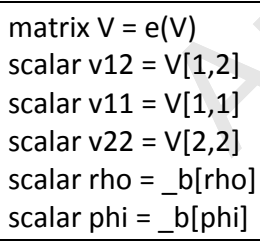

Where the variance of $\hat{\rho}=\mathrm{v} 11$, the variance of $\hat{\phi}=\mathrm{v} 22$ and the covariance between $\hat{\rho}$ and $\hat{\phi}=$ v12 also need to be defined as scalars.

scalar aa $=($ phi*phi $)-(\mathrm{t} 2 * \mathrm{t} 2 * \mathrm{v} 22)$

scalar bb $=(2 * \mathrm{t} 2 * \mathrm{t} 2 * \mathrm{v} 12)-(2 *$ rho*phi)

6

This article is protected by copyright. All rights reserved 
scalar cc $=($ rho*rho $)-(\mathrm{t} 2 * \mathrm{t} 2 * \mathrm{v} 11)$

scalar rad $=$ sqrt $(b b * b b-4 * a a * c c)$

scalar $\operatorname{lm} 1=(-b b+\operatorname{rad}) /(2 * a a)$

scalar $\operatorname{lm} 2=(-b b-\mathrm{rad}) /(2 *$ aa $)$

The roots of the quadratic are then found by first defining the parameters of the quadratic as scalars and then using the solution to the quadratic equations to solve for the roots as $\operatorname{Im} 1$ and $\operatorname{Im} 2$. The result would be a finite $\mathrm{CI}$ if the quadratic has real roots (in this case if rad is not missing).

For a graphical representation of the $100(1-\alpha) \%$ Fieller CI for $\theta$, we first recall the regression results with the restore option on the estimates command then we use the margins command to calculate $\left(\hat{\rho}-\hat{\phi} \theta_{j}\right)$ for different values of $\theta_{j}$ and its corresponding 95\% CIs. In order to obtain a range of values for $\theta_{j}$ we need to use one of the variables in the model (in this case $x_{3}$ ) to act as a proxy for $\theta_{j}{ }^{6}$

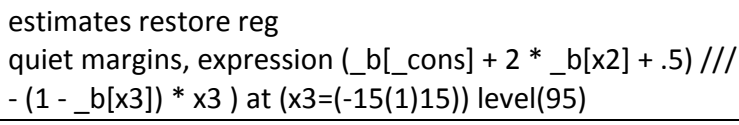

In this case we have considered possible values for $\theta_{j}=-15$ to $\theta_{j}=15$ with increments of 1 . To plot the calculated functions from margins we use the Stata command marginsplot,

\section{Econometric Applications}

In this section, we demonstrate the use of the Fieller method in eight econometric applications where a function of interest is a ratio of parameters or ratio of linear combinations of parameters.

The first example is the interpretation of a dummy variable in terms of changes in a continuous regressor. This technique is related to the slope ratio assay method originally used in pharmacology. The application presented here employs the border phenomenon observed when smoking laws in one state influenced demand for gambling in an adjacent state.

In the second example, we demonstrate how one can determine the turning point in a quadratic specification where the marginal impact of a regressor changes sign. It is frequently the case that non-linearity in a regression is modelled with a quadratic specification. One aspect of this form of specification is that the marginal impact of the regressor is not constant and may change sign within the range of the data. The application considered here is the case of the environmental Kuznets curve that postulates that countries with higher national incomes pollute at decreasing rates when they approach a turning point in the quadratic relationship between pollution and national income. 
The third example examines how the marginal effects of one regressor that is interacted with a second regressor varies with the values of the second variable. In this case we establish the range of values for the second regressor over which the marginal effects of the first variable are significantly greater than zero.

The fourth example, demonstrates how the long-run marginal effects in a dynamic model can be viewed as a ratio. In this example, we use aggregate energy demand that is modelled with a dynamic model to estimate both the short-run and long-run price elasticities. This example also shows how the Stata code can be modified to account for a non-linear estimation model.

The fifth example is another dynamic model where we investigate the "Taylor Rule" for dynamic macro models. In this case the specification is more complex than in the fourth example and demonstrates how the CI for the long-run effect in a model with multiple lags can be estimated.

Our sixth example is the determination of the non-accelerating inflation rate of unemployment (or NAIRU). In this example a Phillips curve relationship is estimated where the NAIRU is found as a ratio of parameter estimates.

In the seventh application, we consider the measure of the willingness-to-pay model that is also related to the $50 \%$ dose model used in pharmacology. Here a binomial dependent variable is modelled as a function of a continuous regressor. The ratio of interest is the value of the continuous regressor that results in a predicted probability of .5. In the willingness-to-pay literature this value is the minimum someone is willing to pay when the dependent variable is the purchase and price is the continuous variable.

In the eighth example, we demonstrate how the structural parameter in an exactly identified system of equations can be estimated via the indirect least squares method which is equivalent to the two-stage least squares approach. This method employs the ratio of parameters for the structural parameter estimation.

\subsection{Parallel-line assay: A compensation interpretation of a dummy variable.}

Finney (1978) considers the slope-ratio assay in which the potency of a test drug in comparison to a standard drug is required. In the assay, amounts of a standard drug are used on some patients while other amounts of a test drug are used on other patients. The assumed model is,

$$
y_{i}=\beta_{0}+\phi x_{i}+\rho d_{i}+\varepsilon_{i},
$$

where $y$ is the response, $x$ is the dosage of the drug, $d$ is an indicator of 0 for standard drug and 1 for test drug and $\rho=(\phi \theta)$. The parameter of interest is $\theta$ which is the ratio of parameters $\theta=\frac{\rho}{\phi}$. This 
can be interpreted as the amount of the standard drug required to give an equivalent response to a unit dose of the test drug.

This method can be generalized to any case where we fit a regression with continuous regressors as well as dummy variables. This technique can provide an interpretation of the value of the coefficient on the dummy variable in terms of the change in the value of a continuous variable. Thus, the usual interpretation of the dummy variable in the units of the dependent variable can be changed to be in the units of one of the independent variables.

Figure 3.1.1 The relationship between the estimated dummy variable parameter $\hat{\rho}$ and the translated version $\hat{\theta}$ in the simple case.

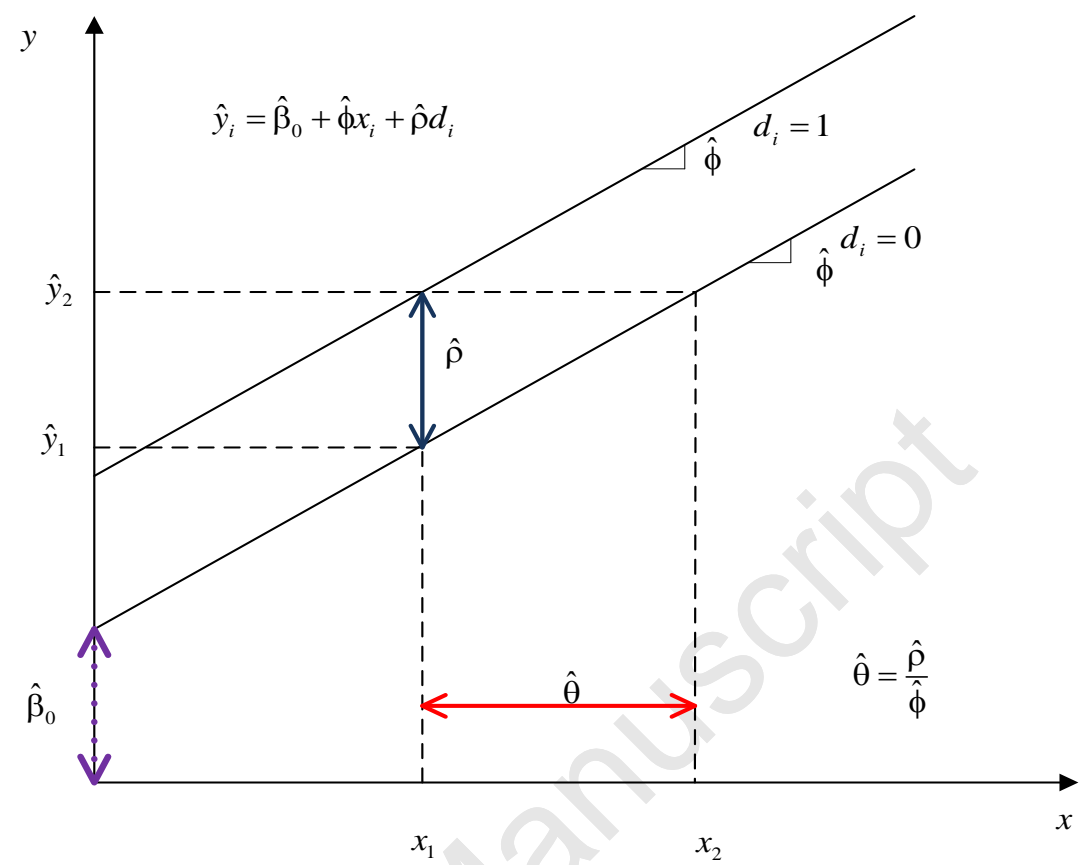

For example, if we fit a model with the specification as in (3.1.1) we would have a predicted relationship as shown in Figure 3.1.1. Where the dummy variable $d$ shifts the linear relationship between $y$ and $x$ by an amount $\hat{\rho}$ for any value of $x$ and the slope of the parallel lines is $\hat{\phi}$. Where we have assumed that all parameters are estimated as positive values. This means that we can interpret $\hat{\rho}$ as the increased amount of $x$ needed to compensate for when $d=0$. Thus, from Figure 3.1.1 if the level of $x$ is $x_{1}$ and $d=1$ then the predicted value of $y$ is given as $\hat{y}_{2}$. However, if the dummy is equal to zero then the predicted value of $y$ is $\hat{y}_{1}$ where $\hat{y}_{1}=\hat{y}_{2}-\hat{\rho}$ and the usual interpretation of $\hat{\rho}$ is in the units of $y$. Alternatively, we can convert the value of $\hat{\rho}$ in terms of the equivalent change in $x$ to have the same effect as the case when the dummy variable is equal to one. This amount is determined by $\hat{\theta}=\frac{\hat{\rho}}{\hat{\phi}}$ which interprets the impact of the dummy in the 
corresponding value of the regressor. However, this translation of units requires the ratio of the estimated values of $\phi$ and $\rho$ which are stochastic terms and thus complicates the interpretation of $\hat{\theta}$ and the construction of its corresponding $\mathrm{CI}$.

In this example, we apply this approach to determine the impact of the proximity to the state border on a smoking ban in gaming areas of licensed premises that was instituted on $1^{\text {st }}$ September 2002 in Victoria, Australia. The objective of this analysis is to determine the increase in the number of EGMs that venues in communities on the border with New South Wales would need to compensate them for the loss in revenue they incurred due to the institution of smoking bans in Victoria and not in New South Wales. Hirschberg and Lye (2010d) investigate the nature of the pattern of falls in local electronic gaming machine (EGM) revenue that occurred due to this ban. The ban had a differential impact on different regions based on their proximity to the border with New South Wales, where there were no smoking bans in effect at the time. Victoria is subdivided into local government areas (LGAs). Using LGA-level annual data, Hirschberg and Lye (2010d) regress the \%change in EGM Expenditure 2002-2003 in \$2002 (\% percentage change in EGM numbers ( $\% \triangle E G M)$, a measure of social-economic status of the LGA (IRSED) and a dummy variable (nborder) that takes the value 1 if the LGA does not border the state of New South Wales. The specification is given in (3.1.2).

$$
\% \Delta \text { EGMExpend }_{i}=\beta_{0}+\beta_{1} I R S E D+\phi \% \Delta E G M_{i}+\text { pnborder }_{i}+\varepsilon_{i}
$$

Table 3.1.1. Impact of Local Smoking Bans regression results (with robust standard errors), where:

fall $=\% \Delta E G M E x p e n d$, irsed $=I R S E D$, cegm $=\% \Delta E G M$, and nborder $=$ nborder.

\begin{tabular}{|c|c|c|c|c|c|c|c|}
\hline \multicolumn{2}{|c|}{ Linear regression } & +1 & & $\begin{array}{l}\text { Numbe } \\
\text { F (3, } \\
\text { Prob } \\
\text { R-squ } \\
\text { Root }\end{array}$ & $\begin{array}{l}\text { f obs } \\
\text { d }\end{array}$ & $\begin{array}{l}= \\
= \\
= \\
= \\
=\end{array}$ & $\begin{array}{r}60 \\
5.25 \\
0.0029 \\
0.2325 \\
3.7914\end{array}$ \\
\hline fall & Coef. & $\begin{array}{c}\text { Robust } \\
\text { Std. Err. }\end{array}$ & t & $P>|t|$ & {$[95 \%$} & Conf. & Interval] \\
\hline $\begin{array}{r}\text { nborder } \\
\text { cegm } \\
\text { irsed } \\
\text { _cons }\end{array}$ & $\begin{array}{r}4.445393 \\
.5034429 \\
-.035189 \\
18.99533\end{array}$ & $\begin{array}{r}1.836437 \\
.1880271 \\
.0112757 \\
11.30684\end{array}$ & $\begin{array}{r}2.42 \\
2.68 \\
-3.12 \\
1.68\end{array}$ & $\begin{array}{l}0.019 \\
0.010 \\
0.003 \\
0.099\end{array}$ & $\begin{array}{r}.76 \\
.126 \\
-.057 \\
-3.65\end{array}$ & $\begin{array}{l}6568 \\
7793 \\
7769 \\
4991\end{array}$ & $\begin{array}{r}8.124218 \\
.8801065 \\
-.012601 \\
41.64565\end{array}$ \\
\hline
\end{tabular}

The regression results are reported in Table 3.1.1. The parameter of interest is $\theta$ which is the ratio of parameters on the dummy for no border (nborder) and the percent change in the number of 
EGMs (cegm) is reported in Table 3.1.2 as 8.830. This implies that the number of EGMs within the LGA would need to increase by this percentage to compensate for the location influence of the smoking bans, when also considering the social-economic status of the LGA. The mean of $\% \triangle E G M$ is -0.208 and it ranges from a minimum of -6.75 and a maximum of 4.69 .

Table 3.1.2 The estimates of $\rho, \phi$ and $\theta$.

\begin{tabular}{|c|c|c|c|c|c|c|}
\hline $\begin{array}{r}\text { rho: } \\
\text { phi: } \\
\text { theta: }\end{array}$ & 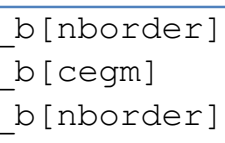 & /_b [ cegm] & & & & \\
\hline fall & Coef. & Std. Err. & z & $P>|z|$ & [95\% Conf. & Interval] \\
\hline rho & 4.445393 & 1.836437 & 2.42 & 0.015 & .846043 & 8.044743 \\
\hline phi & .5034429 & .1880271 & 2.68 & 0.007 & .1349165 & .8719693 \\
\hline theta & 8.829985 & 4.463193 & 1.98 & 0.048 & .0822874 & 17.57768 \\
\hline
\end{tabular}

The appropriate $95 \%$ Delta CI based on the $t$-distribution is [-.111 to 17.771$].^{7}$ Furthermore, the estimated value of $\hat{\theta}=8.83$ is higher than any of the \% changes of EGMs for any observation in the data. We obtained the $95 \%$ Fieller CI as [1.638 to 34.077] from the solution to the quadratic equation as specified in (2.3.4).

The line-plot for the linear combination along with the $\mathrm{CI}$ as defined in (2.3.5) is given in Figure 3.1.2. From the line plot we note that the upper bound is almost double the level of the Delta upper bound. In addition, the upper Fieller bound of more than $34 \%$ would require a change in the number of EGMs to be way beyond a viable number for any year. This implies that to compensate the venues on the border for the advent of a smoking ban to insure they are not disadvantaged more than other Victorian venues, would require an increase of on average 8.83\% more EGMs and to insure compensation with $95 \%$ confidence would require over a $34 \%$ increase. An increase that is way beyond the maximum that was observed over the time and local communities observed up to then. 
Figure 3.1.2 The linear combination $\hat{\rho}-\hat{\phi} \theta$ with the $95 \% \mathrm{CI}$

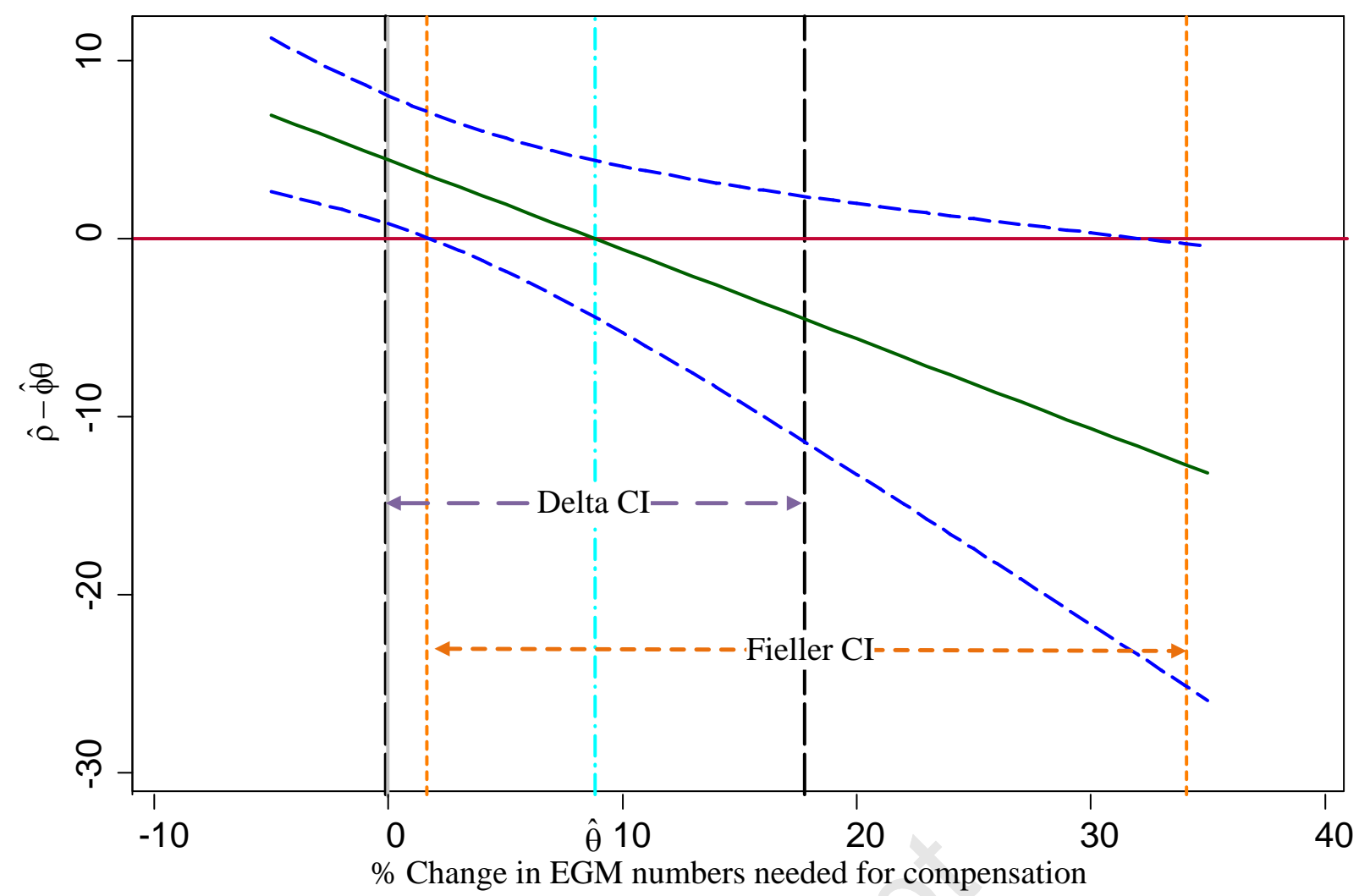

\subsection{The Turning Point: An Environmental Kuznets Curve.}

The estimation of non-linear relationships with a polynomial specification is often used in empirical economic research. Adding a polynomial in a regressor to a model specification will allow the marginal effect of the regressor to vary with the value of the regressor. The most commonly employed polynomial is the quadratic form where the relationship estimated is either a U-shaped or inverted U-shaped curve. In many cases the curvature of the estimated relationship will not imply a sign change in the marginal effect within the range of the data. However, when such a sign change does occur within the range of the regressor values, there is an indication of a turning point. A common example of such a relationship is the Kuznets (1955) curve that proposes that the relationship between income inequality and income, can be represented by an inverted Ushaped curve. Whereby income inequality increases with income then reaches a maximum and then falls with increased income. ${ }^{8}$

The Environmental Kuznets Curve (EKC) hypothesizes an inverted U-shaped relationship between per capita income and pollution. In this case, it is hypothesized that pollution increases with economic growth up to a point where it will start to diminish when an economy reaches a specific income. We use data from Harbaugh et al. (2002) that contains measures of ambient sulfur 
dioxide $\left(\mathrm{SO}_{2}\right)$ concentrations as an indicator of pollution in 102 cities located in 45 countries between 1971 and 1992 to estimate an EKC. The specification is,

$$
\log (S O 2)_{i t}=\beta_{1} G_{i t}+\beta_{2} G_{i t}^{2}+\mathbf{X}_{i t}^{\prime} \boldsymbol{\lambda}+\mu_{i}+v_{i t}
$$

where $S O 2_{i t}$ is the mean annual sulfur dioxide measured by each monitor, $G_{i t}$ is per capita gross domestic product (in 1,000\$) at time $t$ for the country in which monitoring site $i$ is located, $\mathbf{X}_{i t}$ are country and site-specific covariates and year dummy variables and $\mu_{i}$ is a random site-specific effect estimated via random effects and clustered standard errors by site. The turning point is given by:

$$
\theta=\frac{\rho}{\phi}=\frac{-\beta_{1}}{2 \beta_{2}}
$$

Table 3.2.1 Environmental Kuznets Curve regression estimate where: lnmean $=\log (S O 2), \mathrm{GDP}=G, \mathrm{c} . \mathrm{GDP} \# \mathrm{C} \cdot \mathrm{GDP}=G^{2}$, [DENSNAT, TRADE, DEMO $]=\mathbf{X}$, and IYEAR $=\boldsymbol{t}$.

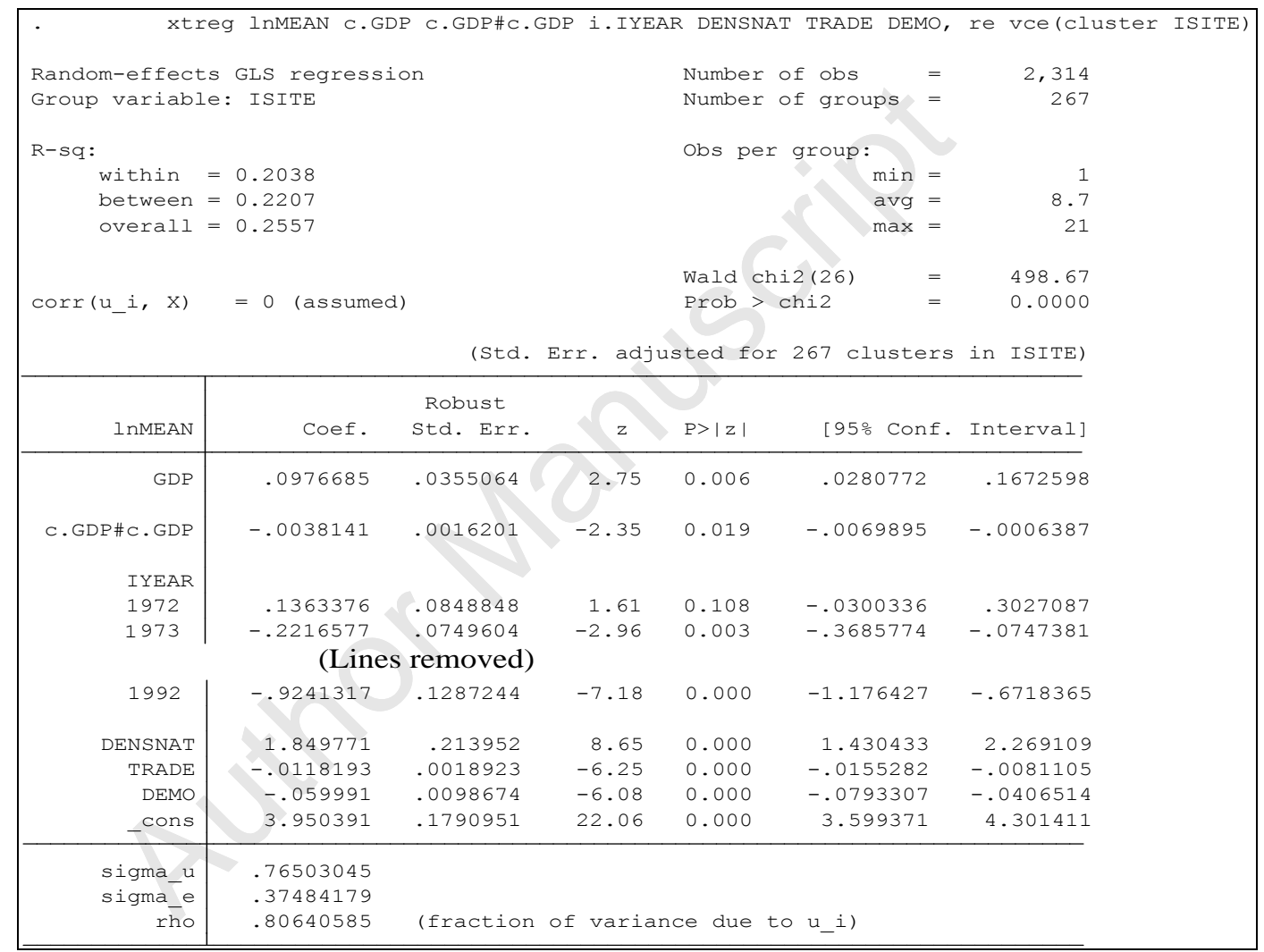

The model was estimated with random effects for each site and fixed effects by year. The $\mathbf{X}_{i t}$ variables include a measure of national trade intensity (TRADE), an index of democratic government (DEMO), and population density (DENSNAT). Indicator variables for each year are also included (i.IYEAR). The $z$-statistics $p$ values are adjusted for the number of clusters defined by site. The Stata 
estimation results are in Table 3.2.1. The ratio gives the estimates of the turning point and the corresponding Delta method CI for the Kuznets curve as obtained by the call to nlcom as shown in Table 3.2.2

Table 3.2.2 Environmental Kuznets Curve estimates of $\rho, \phi$, and $\theta$

\begin{tabular}{|c|c|c|c|c|c|c|}
\hline $\begin{array}{r}\text { rho: } \\
\text { phi: } \\
\text { theta: }\end{array}$ & $\begin{array}{l}\mathrm{b}[\mathrm{GDP}] \\
-2 \star{ }^{\star} \_\mathrm{b}[\mathrm{c} \cdot \mathrm{GDH} \\
\mathrm{b}[\overline{\mathrm{GDP}}] / /(-\end{array}$ & $\begin{array}{l}\text { C.GDP }] \\
\text { *_b }[\mathrm{c} \cdot \mathrm{GDP} \#\end{array}$ & DP ] ) & & & \\
\hline InMEAN & Coef. & Std. Err. & $\mathrm{z}$ & $\mathrm{P}>|\mathrm{z}|$ & [95\% Conf. & Interval ] \\
\hline rho & .0976685 & .0355064 & 2.75 & 0.006 & .0280772 & .1672598 \\
\hline phi & .0076283 & .0032402 & 2.35 & 0.019 & .0012775 & .013979 \\
\hline theta & 12.80351 & 1.748275 & 7.32 & 0.000 & 9.376958 & 16.23007 \\
\hline
\end{tabular}


Figure 3.2.1 The shape of the quadratic relationship and the Fieller interval as determined where the bounds of the first derivative function equals zero.

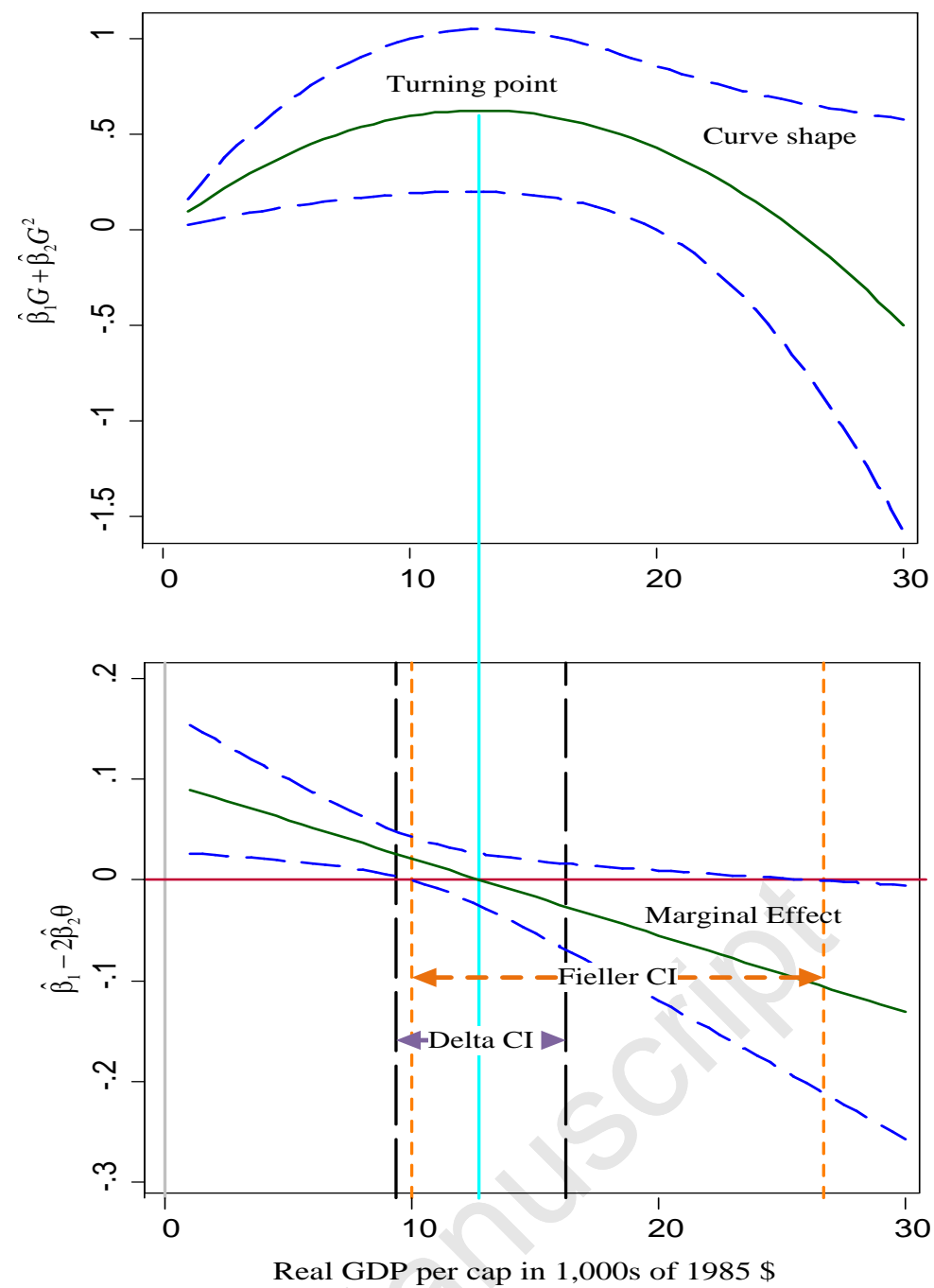

The 95\% Delta CI based on the $t$-distribution for the turning point is 9.361 to 16.246 . To determine the shape of the quadratic relationship we can plot the curve defined by the impact of variations in $G$ as $\hat{\beta}_{1} G+\hat{\beta}_{2} G^{2}$. Figure 3.2.1 is the plot of the implied curve and the first derivative function along with their corresponding $95 \% \mathrm{CI}$.

The Fieller interval as calculated from the solution to the quadratic equation is given as 9.965 to 26.691. From Figure 3.2.1 the wide upper bound of the Fieller as compared to the Delta upper bound corresponds to the flattening out of the upper bound of the $95 \% \mathrm{CI}$ in the top graph of the shape. Note that in this data the GDP per capita has a maximum of 18.095 which would mean that the relationship never would reach the point where the marginal effect of income becomes negative if we use the Fieller interval instead of the Delta interval. This is quite apparent from the curve shape plot in Figure 3.2.1. From this plot we can see that the Delta upper bound at 16.230 is at a 
point where the curve is fairly flat while the Fieller interval would indicate that pollution would not be predicted to fall with higher GDP until it reached 26.691.

\subsection{Interactions: Journal subscriptions as influenced by age and price ${ }^{9}$}

Interaction terms are widely used in econometric models when the influence of two regressors are assumed to have a combination effect. For example, in the model specified by (3.3.1):

$$
y=\beta_{1}+\beta_{2} x+\beta_{3} w+\beta_{4} x w+\beta_{5} v_{1}+\cdots \beta_{k} v_{k-4}+\varepsilon
$$

when both $x$ and $w$ are both continuous variables we define the partial influence function as the marginal effect of a change in $x:^{10}$

$$
\frac{\partial \mathrm{E}(y / x, w)}{\partial x}=\beta_{2}+\beta_{4} w
$$

If $x$ is a dummy variable and $w$ is a continuous variable, the difference in the regression equation when $x=1$ and when $x=0$ is defined as:

$$
\frac{\Delta \mathrm{E}(y / x, w)}{\Delta x}=\beta_{2}+\beta_{4} w
$$

However, both the expressions in (3.3.2) and (3.3.3) introduce the difficulty of an appropriate choice of the other regressor at which to evaluate these margins.

One approach is to set the value of this other regressor to a value such as the mean and then make the computation. Alternatively, one can determine at what value of the other regressor do these definitions go to zero or change sign. In specification (3.3.1) this would be the value of $w$ that results in $\frac{\partial \mathrm{E}(y / x, w)}{\partial x}=c$ or $\frac{\Delta \mathrm{E}(y / x, w)}{\Delta x}=c$ where typically $c=0$ or some other value. From (3.3.2) and (3.3.3) we would find this value as $w^{*}=\frac{c-\beta_{2}}{\beta_{4}}$ and is estimated by $\hat{w}^{*}=\frac{c-\hat{\beta}_{2}}{\hat{\beta}_{4}}$ where $\hat{\beta}_{i}$ are the OLS estimates of the $\beta_{i}, \mathrm{i}=2,4$. The question of interest is whether the value of $\hat{w}^{*}$ would ever be close to $w^{*}$ or not. We propose that this may be investigated via the use of the partial influence function that maps how $\frac{\partial \mathrm{E}(y / x, w)}{\partial x}$ or $\frac{\Delta \mathrm{E}(y / x, w)}{\Delta x}$ vary with values of $w$.

For an example of the application of the partial influence function we use an analysis from Stock and Watson (2003, pg. 227) in which they estimate the relationship between the number of subscriptions to a journal at US libraries $(Y)$ and its library subscriptions price. The data are for the year 2000 and cover 180 economics journals. Price is measured in prices per citation which is used as a proxy variable for dollars per idea. Other explanatory variables include the age of journal (Age) and the number of characters printed per year in the journal (Char) as a measure of the 
content of the journal. Here we interact the price variable with age to allow for the price elasticity to vary with the age of the journal. The regression equation is specified as:

$$
\begin{aligned}
\log (Y) & =\beta_{1}+\beta_{2} \log (\text { Age })+\beta_{3} \log (\text { Price }) \\
& +\beta_{4}(\log (\text { Price }) \times \log (\text { Age }))+\beta_{5} \log (\text { Char })+\varepsilon
\end{aligned}
$$

The regression results are reported in Table 3.3.1:

Table 3.3.1: Estimated regression results for Library Journal Subscriptions. Where: $\operatorname{loclc}_{\mathrm{C}} \log (Y), \operatorname{lage}=\log ($ Age $), \operatorname{lprice}=\log ($ Price $), \operatorname{lpla}=(\log ($ Price $) \times \log ($ Age $))$ and lchar $=\log ($ Char $)$.

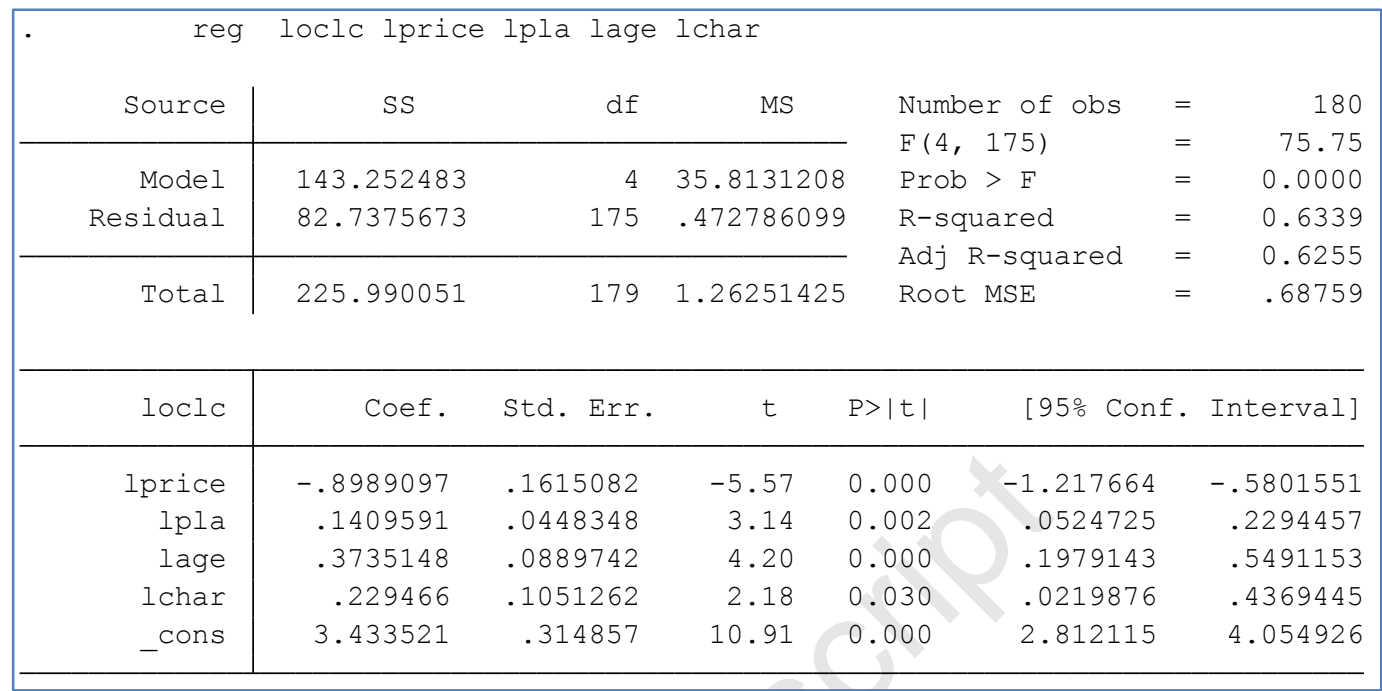

Based on these estimates, we find that the estimated price elasticity is defined as:

$$
-\hat{\beta}_{2}+\hat{\beta}_{4} \times \log (\text { Age })=-0.899+0.141 \times \log (\text { Age })
$$

(3.3.5) implies that the price elasticity of a journal will be smaller in magnitude (less negative) with age - in other words: libraries are less sensitive to price changes for journals that have been around longer. The ratio $\left({ }^{0.899} / .141\right)$ defines the value of $\log ($ Age $)$ at which $-0.899+0.141 \times \log ($ Age $)=0$.

Figure 3.3.1 plots the estimated price elasticity and its corresponding confidence bound. Since the actual values of $A g e$ in the data has a minimum value of $4(\log (A g e)=0.60)$ and a maximum value of $156(\log (\mathrm{Age})=5.05)$ we conclude from Figure 3.3.1 that the price elasticity is significantly different from 0 for all the journal ages in this data set.

It is common practice to report a summary price elasticity such as the value of the mean of all the price elasticities which in this case equals -0.439 . To find the $95 \%$ Delta and Fieller intervals for this case define $\hat{\theta}=\frac{\hat{\rho}}{\hat{\phi}}=\frac{-.439-\beta_{2}}{\beta_{4}}$. We compute this value of $\hat{\theta}$ and the corresponding $95 \% \mathrm{CI}$ using the Delta method to find the bounds from 2.667 to $3.853 .{ }^{11}$ From Table 3.3.2 we find the 17 This article is protected by copyright. All rights reserved 
Delta CI bounds limits are when $A g e$ is less than $14.5(\log (A g e)=2.67)$ or $A g e$ is greater than 46.8 $(\log ($ Age $)=3.85)$.

Table 3.3.2 The Delta estimate for the ratio of rho and phi. (m_elast is the mean of the elasticities $(-0.439))$

\begin{tabular}{|c|c|c|c|c|c|c|}
\hline loclc & Coef. & Std. Err. & z & $\mathrm{P}>|\mathrm{z}|$ & [95\% Conf. & Interval] \\
\hline rho & .459526 & .1615082 & 2.85 & 0.004 & .1429758 & .7760762 \\
\hline phi & .1409591 & .0448348 & 3.14 & 0.002 & .0530844 & .2288337 \\
\hline theta & 3.259996 & .2990973 & 10.90 & 0.000 & 2.673776 & 3.846216 \\
\hline
\end{tabular}

Figure 3.3.1: Price Elasticity Function for the Demand for Economics Journals

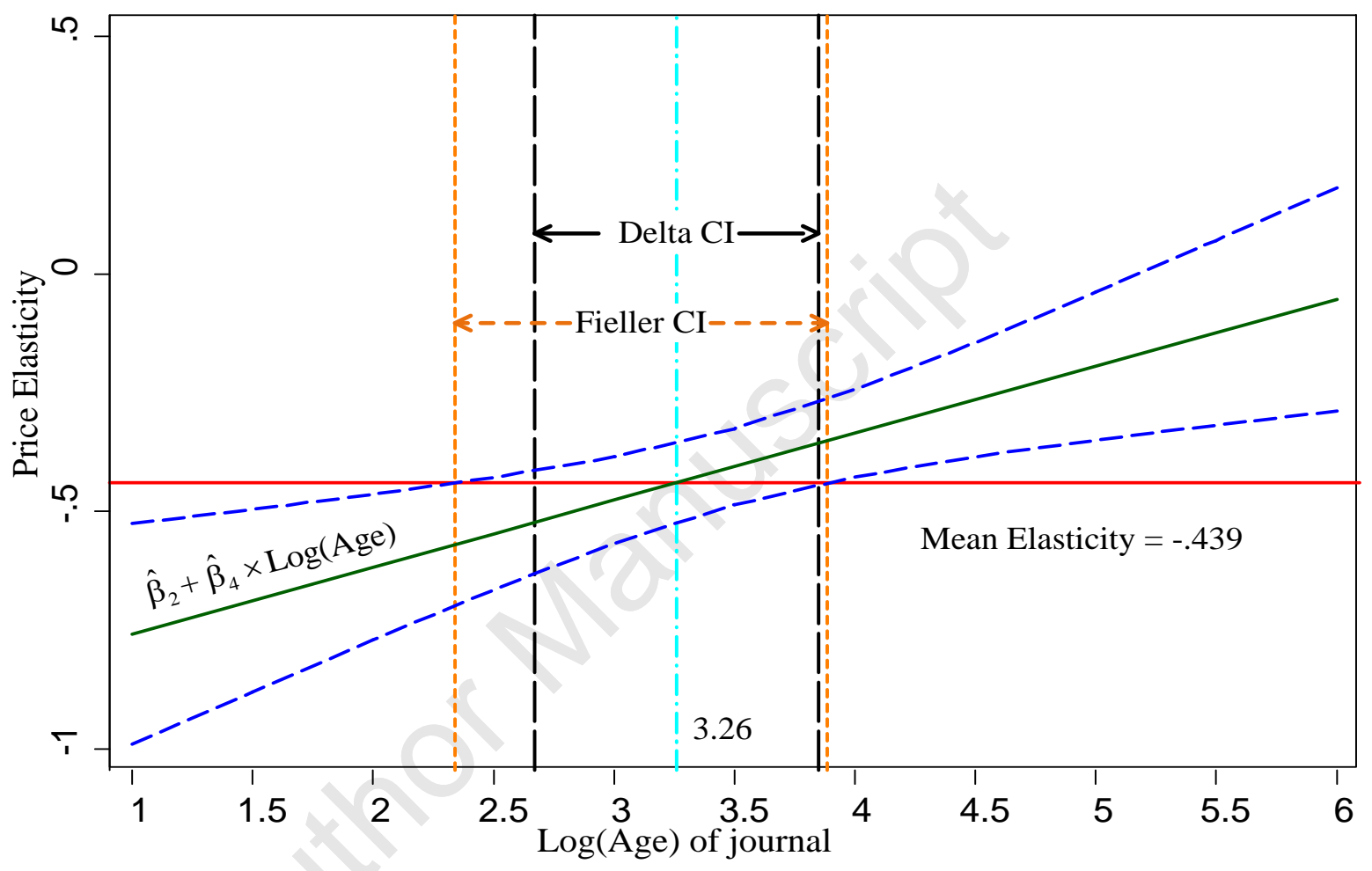

The $95 \%$ Fieller CI can be computed based on the solution to (2.3.4) to be 2.342 to 3.886 which results in lower values for both the lower bound and upper bound. These values can be found from Figure 3.3.1 where the 95\% confidence interval of the price elasticity plotted as a function of age, cuts the line at the mean elasticity of -.439. 


\subsection{The Long-run: A non-linear specification for energy demand}

Dynamic model specifications are widely employed for the analysis of time-series data. A dynamic specification includes a lag or multi-period lags of the dependent variable as regressors. One of the consequences of this type of model is the need for the interpretation of the parameter estimates of other regressors in relation to the estimated coefficients on the lagged dependent variables.

A simple dynamic specification with one lag is defined by:

$$
y_{t}=\lambda y_{t-1}+\beta x_{t}+\varepsilon_{t}
$$

In this model $y_{t}$ is the dependent variable at time $t, x_{t}$ is an explanatory variable at time $t$ and $\varepsilon_{t}$ is the error at time $t .{ }^{12}$ In the long-run it is assumed that the values of $y$ will tend to a specific value (designated as $\tilde{y}$ ). Thus, $y_{T}=y_{T-1}=\tilde{y}$ when $t$ reaches the long-run at time $T$. Under the assumption of a stable process ( that $|\lambda|<1)$, the long-run model can be written as $\tilde{y}-\lambda \tilde{y}=\beta x_{t}+\varepsilon_{t}$ or $\tilde{y}=(1-\lambda)^{-1}\left(\beta x_{t}+\varepsilon_{t}\right)$ and the long-run marginal effect of $x$ on $\tilde{y}$ or $\frac{\partial \tilde{y}}{\partial x}$ is defined as a ratio $\theta=\frac{\rho}{\phi}=\frac{\beta}{1-\lambda}$. Since the estimate of $\lambda$ in most economic applications is positive we find that the marginal impact in the long-run of a change in $x$ is usually greater in magnitude than the short-run marginal effect estimated by $\beta$.

Bernard et al (2007) employ a non-linear dynamic specification in their estimate of an energy demand model for the commercial sector of the Québec economy using annual data from 1962 to 2000. Sector specific total energy demand measured in terajoules (TE) is modelled as a function of its own lag, real energy price $(P E)$ real income $(I N)$, heating degree days as a measure of heating demand caused by low temperatures $(H D D)$ and an error term $\varepsilon$. To ensure that temperature effects for year $t$ are restricted to year $t$, the coefficient of $H D D_{\mathrm{t}-1}$ is set to $-\lambda \pi_{3}$. The model is specified in (3.4.2).

$$
\begin{aligned}
\log \left(T E_{t}\right)=\pi_{0} & +\lambda \log \left(T E_{t-1}\right)+\pi_{1} \log \left(P E_{t}\right)+\pi_{2} \log \left(I N_{t}\right) \\
& +\pi_{3}\left[\log \left(H D D_{t}\right)-\lambda \log \left(H D D_{t-1}\right)\right]+\varepsilon_{t}
\end{aligned}
$$

where the long run price elasticity is: $\theta=\frac{\rho}{\phi}=\frac{\pi_{1}}{1-\lambda}$.

We estimate the model and report the results in Table 3.4.1. From these results, we find that the model appears to fit quite well. The estimated parameter on the lagged dependent variable is .401 , and the short-run price elasticity estimate is -.319 . Table 3.4.2 provides the estimates of the 
long-run price elasticity with the corresponding CI based on the Delta method. From this table we find the estimated long-run price elasticity is -.532 . This result would be in keeping with the usual assumption that the long-run estimates are greater in magnitude than the short-run case.

Table 3.4.1: Energy demand model for the commercial sector of the Québec economy as estimated via non-linear least squares. Where:

logteg $=\log \left(T E_{t}\right), \log l$ teg $=\log \left(T E_{t-1}\right), \operatorname{logep}=\log \left(P E_{t}\right), \log \log =\log \left(I N_{t}\right)$, $\operatorname{loghdd}=\log \left(H D D_{t}\right), \log \operatorname{lhdd}=\log \left(H D D_{t-1}\right), \mathrm{b} 0=\hat{\pi}_{0}, \mathrm{~b} 1=\hat{\lambda}, \mathrm{b} 2=\hat{\pi}_{1}, \mathrm{~b} 3=\hat{\pi}_{2}$, and $\mathrm{b} 4=\hat{\pi}_{3}$.

\begin{tabular}{|c|c|c|c|c|c|}
\hline \multicolumn{6}{|l|}{ 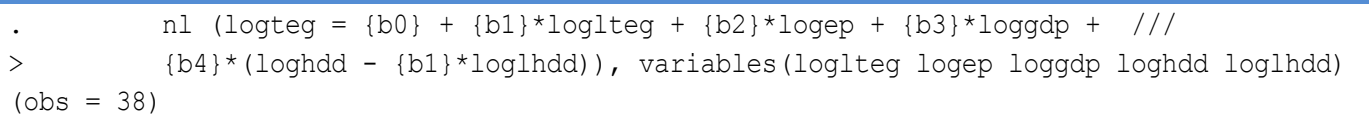 } \\
\hline Source & SS & $d f$ & MS & \multirow{2}{*}{$\begin{array}{ll}\text { Number of obs } & = \\
\text { R-squared } & =\end{array}$} & \multirow{2}{*}{$\begin{array}{r}38 \\
0.9504\end{array}$} \\
\hline Model & 1.9148677 & 4 & .47871692 & & \\
\hline Residual & .10000236 & 33 & .003030374 & Adj R-squared = & 0.9444 \\
\hline Total & 2.01487 & 37 & .054455947 & Res. dev. & -117.8863 \\
\hline logteg & Coef. & Std. Err. & t & [95\% Conf. & Interval] \\
\hline$/ \mathrm{bo}$ & 1.83286 & .411209 & 4.46 & .9962492 & 2.669471 \\
\hline$/ \mathrm{b} 1$ & .4006196 & .0966556 & 4.14 & .2039723 & .5972669 \\
\hline$/ \mathrm{b} 2$ & -.3187478 & .076197 & -4.18 & -.4737719 & -.1637238 \\
\hline /b3 & .5463033 & .1223594 & 4.46 & .2973612 & .7952454 \\
\hline$/ \mathrm{b} 4$ & .4603591 & .1698961 & 2.71 & .114703 & .8060152 \\
\hline
\end{tabular}

Table 3.4.2 The Delta CI for the long-run price elasticity

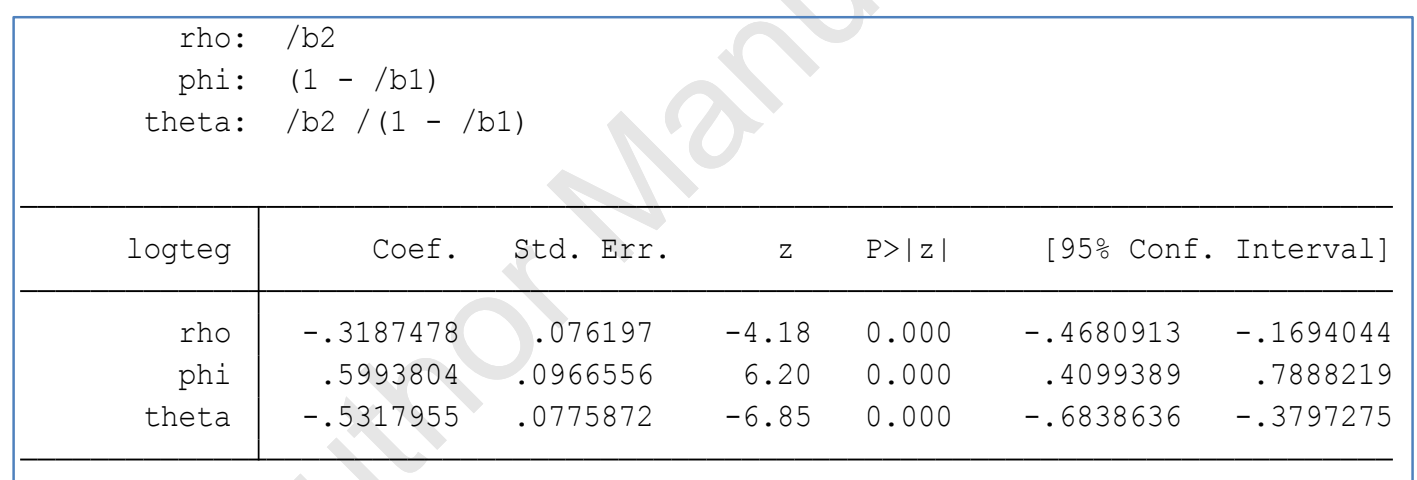

The Fieller interval can be determined from Figure 3.4.1 or from the solution to the quadratic equation as -.681 to -.360 - both would indicate that the lower bound is lower but the upper bound is higher than the Delta result. ${ }^{13}$ However, since the value of $\hat{\phi}$ has a very large t-statistic of -6.20 and the correlation between the numerator and denominator is -.802 (large and the same sign as the long-run elasticity) it is not surprising that the two methods agree on the CI so closely. ${ }^{14}$ 
Figure 3.4.1 The Graphic representation of the Fieller CI

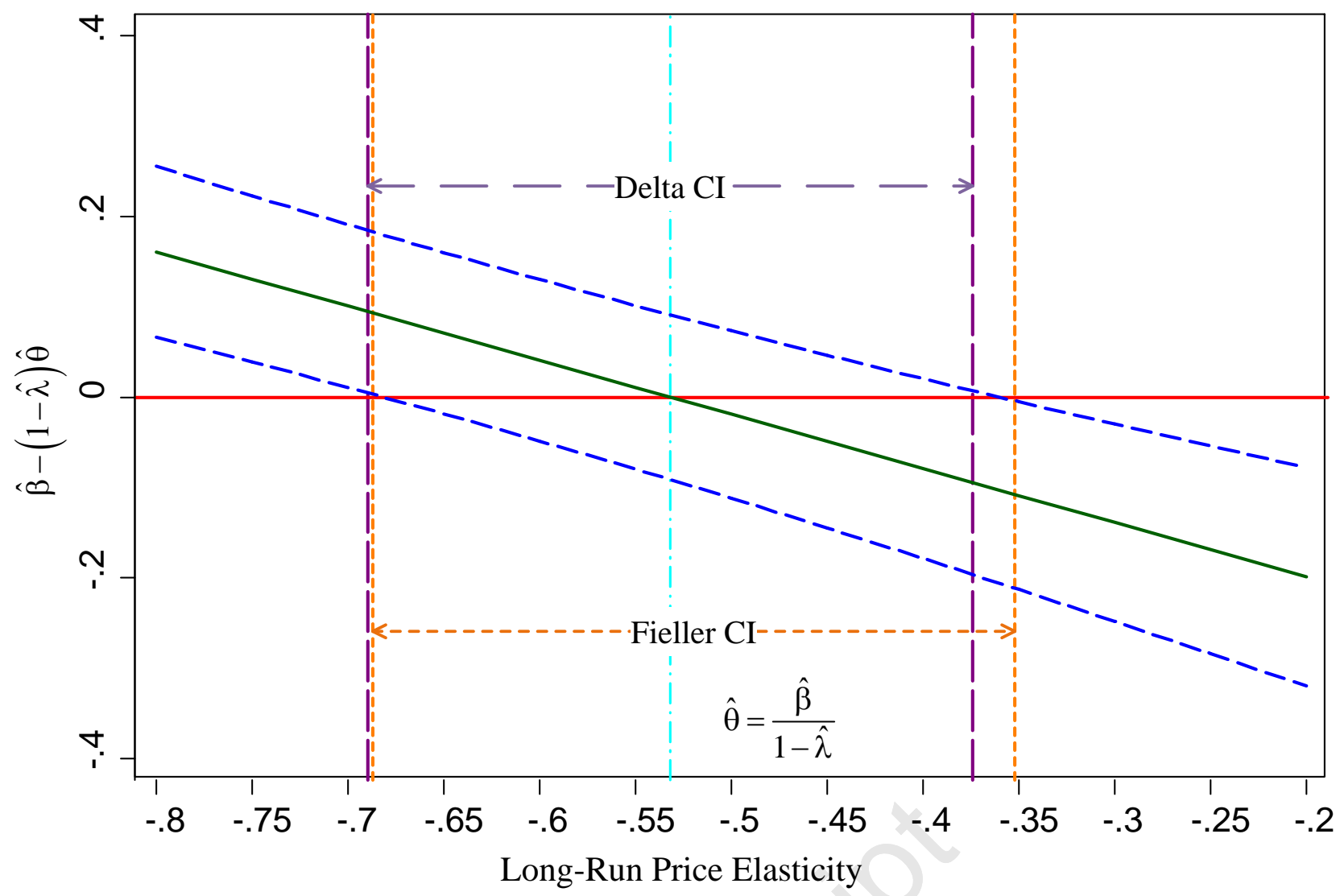

\subsection{A Multiple period lag model: Taylor Rule Coefficients}

Another application of a long run effect is the estimation of Taylor Rules (see Taylor (1993)) with interest rate smoothing and forward looking data. Coibion and Gorodnichenko (2011) estimate a dynamic specification with one and two periods lagged dependent variables. The logic for determining the long-run impact of the other regressors is the same as in the case of a one-period lag dynamic specification except in this case we have need to consider the sum of the parameters on two lagged dependent variables. The specification is of the form,

$$
r_{t}=\alpha+\lambda_{1} r_{t-1}+\lambda_{2} r_{t-2}+\beta_{1} E_{t} \pi_{t+j}+\beta_{2} E_{t} g y_{t+j}+\beta_{3} E_{x} x_{t+j}+\varepsilon_{t}
$$

where $r_{t}$ is the nominal interest rate in quarter $t, g y_{t+j}$ is output growth predicted $j$ quarters in the future from quarter $t, \pi_{t+j}$ is inflation predicted $j$ quarters in the future from quarter $t$, and $x_{t+j}$ is the output gap predicted $j$ quarters in the future from quarter $t$. The long-run parameter of interest is defined as: $\theta=\frac{\rho}{\phi}=\frac{\beta_{1}}{1-\lambda_{1}-\lambda_{2}}$. The interpretation of this parameter is the long run response of interest rates to a permanent 1-percentage point increase in inflation. The model is estimated using realtime data using the Greenbook forecasts from the Philadelphia Federal Reserve Bank of future macroeconomic variables. ${ }^{15}$ In Table 3.5.1 we report the results of their baseline model for the 21 
1983-2002 period where the standard errors are estimated using a Newey-West error specification with 5 lags.

Table 3.5.1 Estimates of the Taylor Rule dynamic regression, where: $\mathrm{ffr}=r_{t}, \mathrm{ffr} 1=r_{t-1}, \mathrm{ffr} 2=r_{t-2}, \mathrm{pi} \_\mathrm{tp}=E_{t} \pi_{t+j}$, gry_tp $=E_{t} g y_{t+j}$, and gap_t03 $=E_{x} x_{t+j}$.

\begin{tabular}{|c|c|c|c|c|c|c|c|}
\hline $\begin{array}{l}\text { Regression wit } \\
\text { maximum lag: } 5\end{array}$ & Newey-West & \multicolumn{2}{|c|}{ standard errors } & $\begin{array}{l}\text { Number } \\
\text { F ( 5, } \\
\text { Prob }\end{array}$ & $\begin{array}{c}\mathrm{f} \text { obs } \\
1\end{array}$ & 52) $\begin{aligned}= \\
=\end{aligned}$ & $\begin{array}{r}158 \\
2015.17 \\
0.0000\end{array}$ \\
\hline $\mathrm{ffr}$ & Coef. & $\begin{array}{l}\text { Newey-West } \\
\text { Std. Err. }\end{array}$ & t & $P>|t|$ & {$[95 \%$} & Conf & Interval] \\
\hline $\operatorname{lffr} 1$ & 1.279632 & .0834952 & 15.33 & 0.000 & 1.11 & 4671 & 1.444593 \\
\hline $\operatorname{lffr} 2$ & -.3393612 & .0814875 & -4.16 & 0.000 & -.500 & 3556 & -.1783669 \\
\hline$p i \_t p$ & .1513119 & .0588523 & 2.57 & 0.011 & .035 & 0378 & .2675861 \\
\hline gry_tp & .1302064 & .0339801 & 3.83 & 0.000 & .063 & 0721 & .1973408 \\
\hline gap_t03 & .0354846 & .0129752 & 2.73 & 0.007 & .009 & 8496 & .0611196 \\
\hline _cons & -.3927445 & .1483813 & -2.65 & 0.009 & -.685 & 9006 & -.0995885 \\
\hline
\end{tabular}

The estimates of $\theta, \rho$ and $\phi$ are listed in Table 3.5.2.

Table 3.5.2 The estimates of of $\theta, \rho$ and $\phi$.

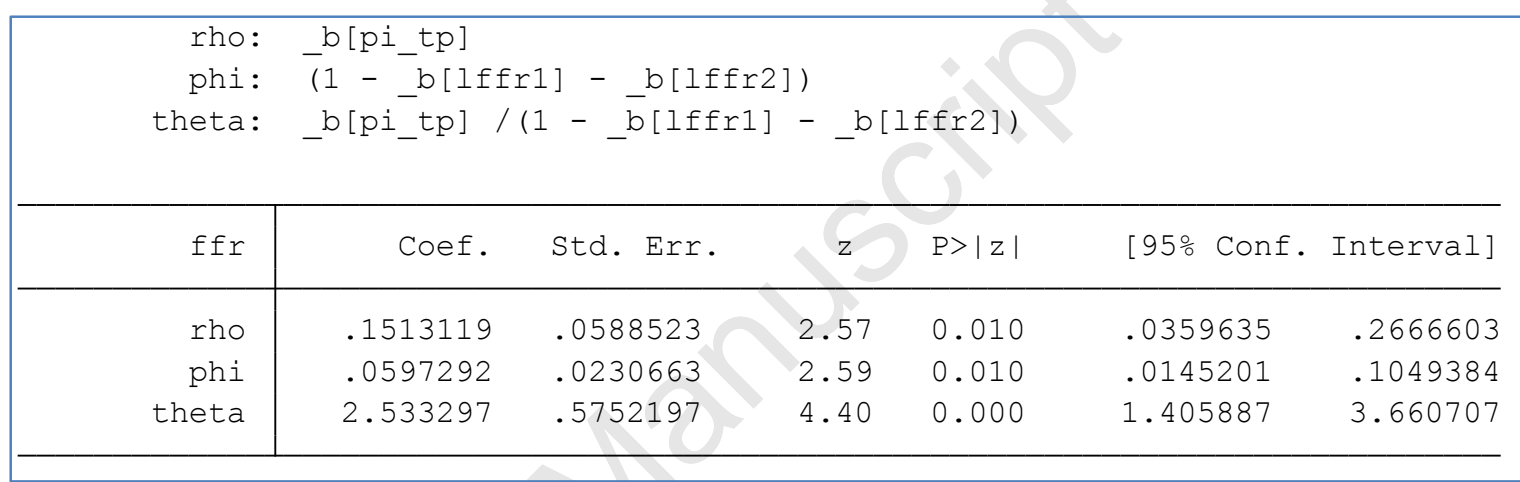

Using the $t$-distribution and the estimated standard error given in Table 3.5.2 we find that the $95 \%$ Delta $\mathrm{CI}$ is 1.397 to 3.670 , and $\hat{\theta}=2.533$. To plot the linear combination of the parameters that can be used to define the Fieller interval we plot $\hat{\beta}_{1}-\theta\left(1-\hat{\lambda}_{1}-\hat{\lambda}_{2}\right)$ with different values of $\theta$ to find where this linear combination is equal to zero along with the $95 \% \mathrm{CI}$ as shown below in Figure 3.5.1. Note that the Fieller CI has both lower and higher limits than the corresponding Delta CI. The Fieller CI is defined as: 1.265 to 4.971. 
Figure 3.5.1 The Fieller and Delta CIs for the long-run

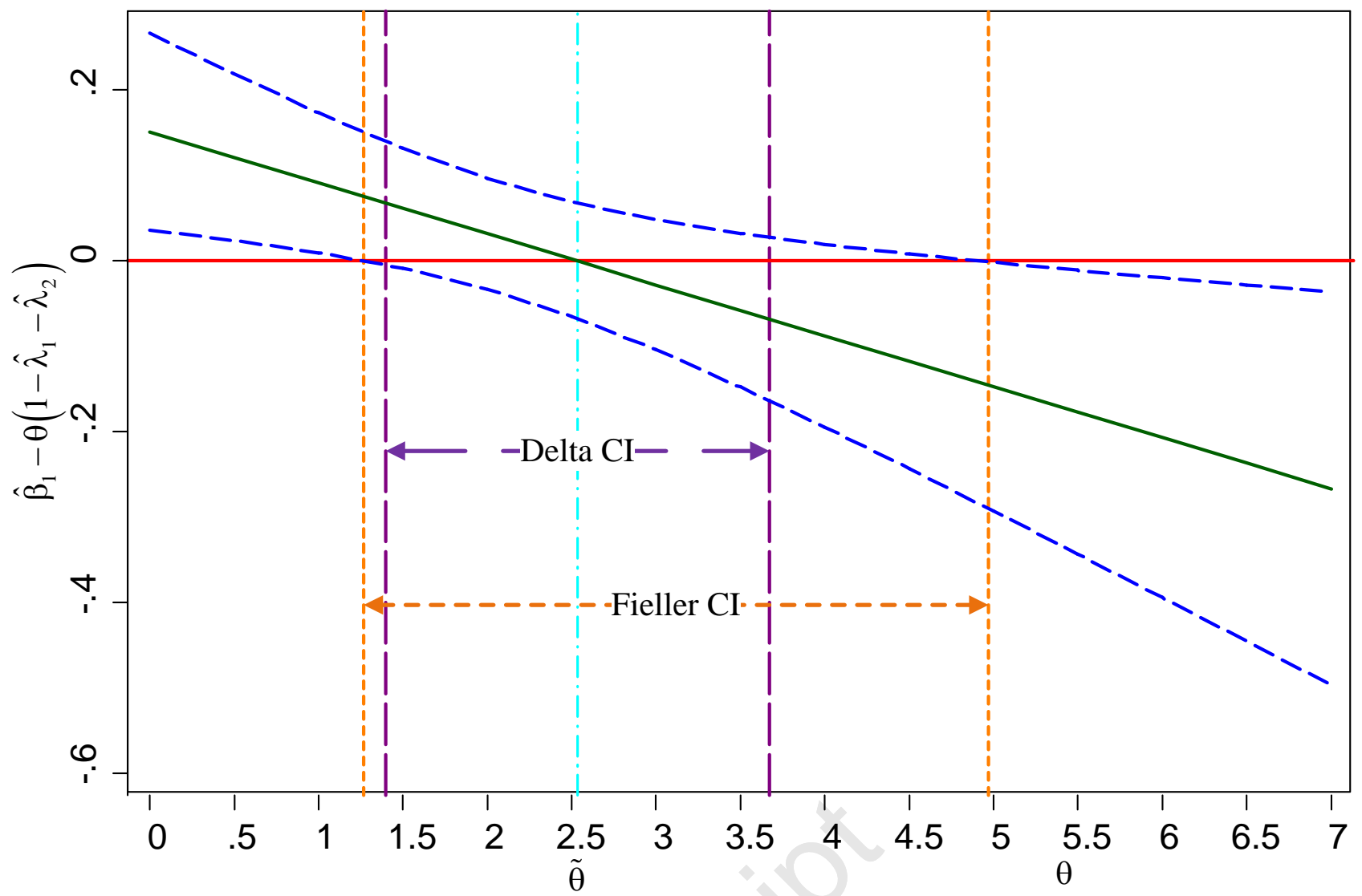

\subsection{The Non-Accelerating Inflation Rate of Unemployment (NAIRU) 16}

The Phillips (1958) Curve is the proposed relationship between inflation and unemployment. The non-accelerating inflation rate of unemployment (NAIRU see Modigliani and Papademos 1975 ) is a version of the natural rate of unemployment designed to account for expectations. Typically, these models specify a measure of inflation as the dependent variable with unemployment as a regressor along with other regressors to account for unexpected changes in prices. Thus, allowing one to solve for the implied level of unemployment when inflation is equal to zero and the unexpected changes are zero as well by the ratio of the intercept to minus the parameter on unemployment.

For example, Gruen et al (1999) use Australian data to estimate a model of the rate of wage inflation, measured by the rate of change of unit labour costs, as a function of the level of unemployment, the change in the rate of unemployment and the expected rate of inflation. They also choose to model annual movements and include a lagged dependent variable to determine the long run process. We write this specification as: 


$$
\begin{aligned}
\Delta_{4} \ln U L C_{t}-\Delta_{4} \ln P_{t-1} & =\alpha_{0}+\alpha_{1}\left(\Delta_{4} \ln P_{t}^{*}-\Delta_{4} \ln P_{t-1}\right)+\alpha_{2} U_{t} \\
& +\alpha_{3} \Delta U_{t-1}+\alpha_{4}\left(\Delta_{4} \ln U L C_{t-1}-\Delta_{4} \ln P_{t-2}\right) \\
& +\alpha_{5}\left(\Delta_{4} \ln U L C_{t-1}-\Delta_{4} \ln U L C_{t-4}\right)+\varepsilon_{t}
\end{aligned}
$$

where $U L C=$ unit labour costs per person, and is equal to wages per person divided by non-farm productivity per person; $P=\mathrm{CPI}, P^{*}=$ expected price level; $U=$ rate of unemployment; $\Delta=1$ quarter change; and $\Delta_{4}=4$ quarter (yearly) change. An estimate of the NAIRU, (the value of $U$ when $\Delta_{4} \ln U L C_{t}-\Delta_{4} \ln P_{t-1}=0$ ), is given as the ratio of the intercept and minus the parameter on the unemployment level: $\hat{\theta}=\frac{\hat{\rho}}{\hat{\phi}}=\frac{\hat{\alpha}_{0}}{-\hat{\alpha}_{2}}$, where $\hat{\alpha}_{0}, \hat{\alpha}_{2}$ are the corresponding estimates of the parameters in (3.6.1). We report the results in Table 3.6.1.

Table 3.6.1: Phillips curve estimates for Australia 1985:1-2003:4 using Newey-West standard errors with 4 lags where: $\mathrm{y}=\Delta_{4} \ln U L C_{t}-\Delta_{4} \ln P_{t-1}, \mathrm{x} 2=\Delta_{4} \ln P_{t}^{*}-\Delta_{4} \ln P_{t-1}, \times 3=U_{t}, x 4=\Delta U_{t-1}$, ×5 $=\Delta_{4} \ln U L C_{t-1}-\Delta_{4} \ln P_{t-2}$, and ×6 $6 \Delta_{4} \ln U L C_{t-1}-\Delta_{4} \ln U L C_{t-4}$.

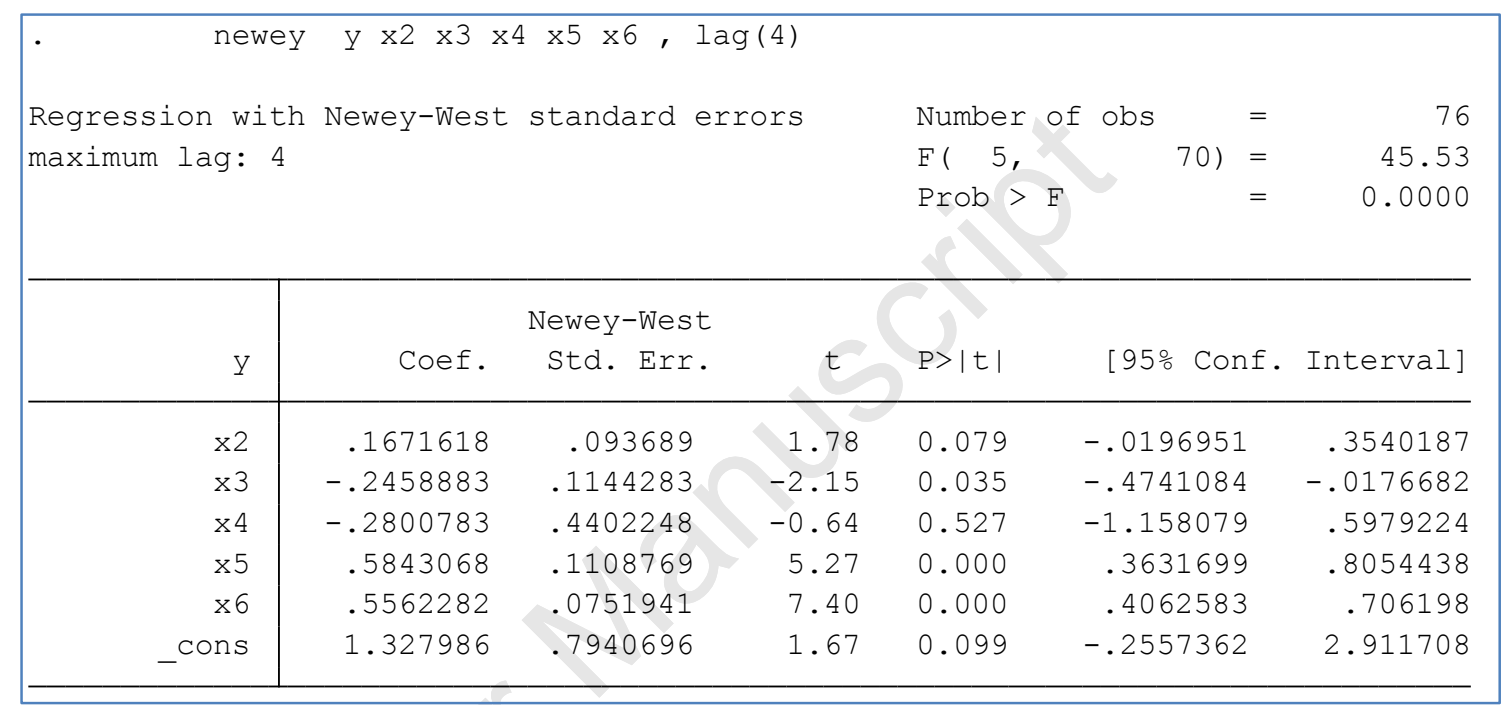

The results of the Delta CI are listed in Table 3.6.2 and from this table we find that the estimated value of the NAIRU $(\hat{\theta})$ is determined as 5.401 with a $95 \%$ CI based on the $t$-distribution of 3.528 to 7.274 . This implies that we could reject the null hypothesis that the NAIRU is equal to zero.

Table 3.6.2: The estimate of the NAIRU with the corresponding Delta CI.

\begin{tabular}{r|rrrrrr}
\hline$y$ & Coef. & Std. Err. & $z$ & P >|z| & [95\% Conf. Interval] \\
\hline rho & -1.327986 & .7940696 & -1.67 & 0.094 & -2.884334 & .2283621 \\
phi & -.2458883 & .1144283 & -2.15 & 0.032 & -.4701637 & -.0216129 \\
theta & 5.400769 & .9391422 & 5.75 & 0.000 & 3.560084 & 7.241454 \\
\hline
\end{tabular}


However, this is a case where the Fieller CI implies a very different result from the Delta estimate. In this case the $95 \%$ Fieller interval is estimated as -13.137 to 6.767 . Thus, we have estimated that the higher limit is less than the corresponding Delta one while the lower limit is much lower and would imply that a test of the hypothesis that the NAIRU is equal to zero could not be rejected at the $95 \%$ level based on the Fieller interval. Note that this would also be true for the $90 \%$ interval when $\alpha=.10$ (in this case the Fieller interval would be .073 to 6.556 ). The contrast between the Delta and Fieller CIs is most evident from the line plot shown in Figure 3.6.1.

Figure 3.6.1 The 95\% Fieller and Delta CIs for the NAIRU.

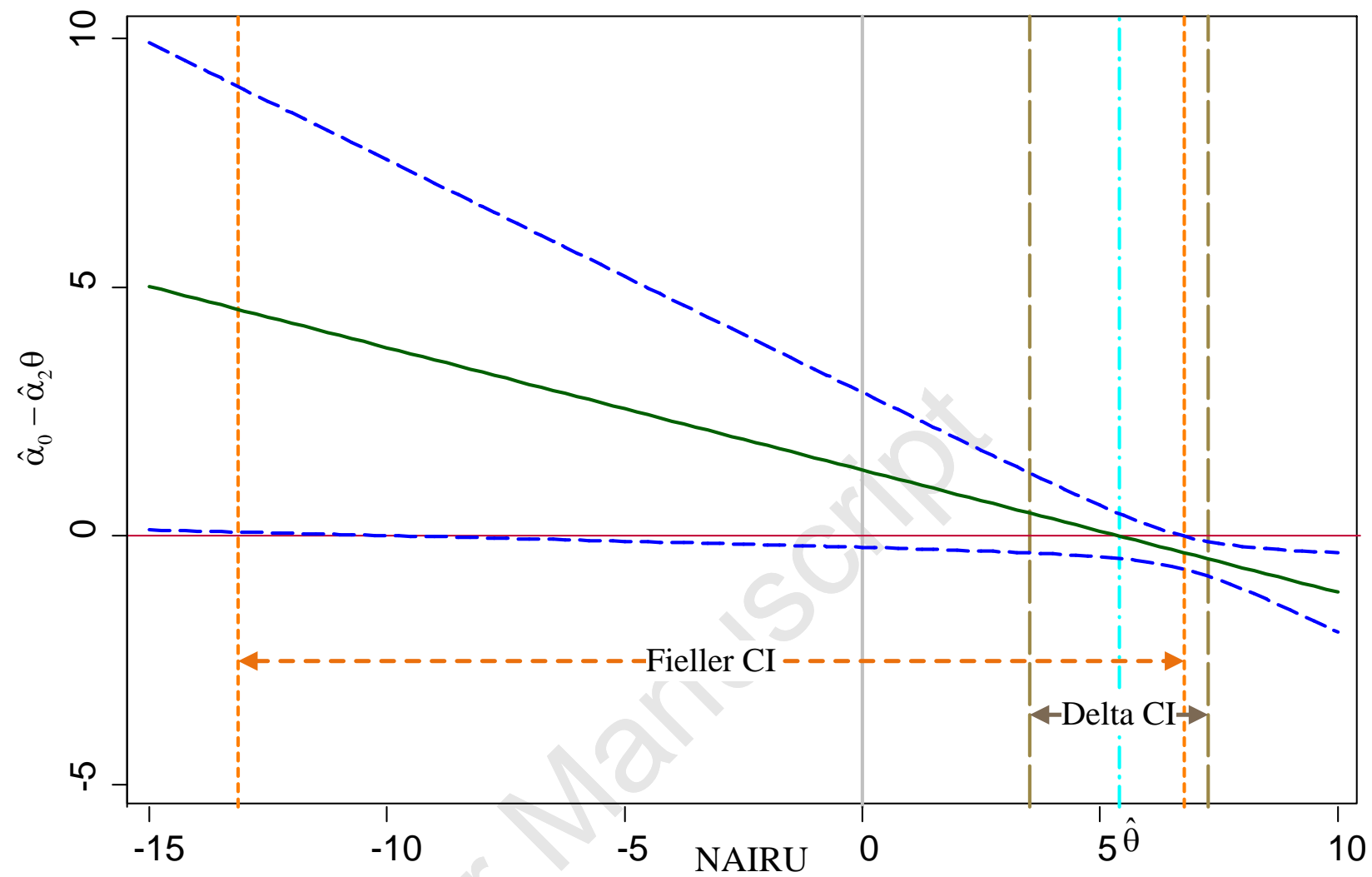

\subsection{The 50\% dose and Willingness-to-Pay.}

The 50\% dose problem was the original application that Fieller (1944) considered when he first investigated the CI of the ratio of parameter estimates. In the pharmacology literature, the 50\% dose refers to the limits of the dose of a drug that would result in a greater than $50 \%$ chance that it would act on the subject. In the economics literature, the willingness-to-pay is defined as the maximum price someone will pay to purchase the product or service (Hole 2007 presents an extensive review). In this type of analysis, the data consists of a dichotomous response to a continuous stimulus $-i$. e. the drug dose or a price. Typically, this problem is modelled by a 
probability of occurrence/purchase using a binary dependent variable model such as a logit or probit estimator,

$$
P\left(Y_{t}=1\right)=\alpha+\gamma X_{t}+\sum_{i=1}^{k} \beta_{i} W_{i t}+\varepsilon_{t}
$$

where $X$ is the variable dose/price and the $W$ 's are other covariates. The question posed by the $50 \%$ dose is: What value of $X$ (designated as $\theta$ ) makes this relationship true?

$$
.5=G\left(\alpha+\gamma \theta+\sum_{i=1}^{k} \beta_{i} W_{i t}+\varepsilon_{t}\right)
$$

Where $G(\bullet)$ is the transformation of the linear function to the probability ( equal to 1 for the linear probability model, the cdf for the logit and probit). Solving for $\theta$ we have the ratio given by:

$$
\theta=\frac{\rho}{\phi}=\frac{G^{-1}(.5)-\left(\alpha+\sum_{i=1}^{k} \beta_{i} W_{i t}\right)}{\gamma}
$$

The ratio can be evaluated at specific values of $W$ for cases of interest or at the means of these other regressors.

As an example, we use data on the survivors of the Titanic disaster where the dose/price is the fare paid for the trip in pounds (see Frey et al 2010) and the survival of the disaster ${ }^{18}$

$$
P(\text { survive })=\alpha+\gamma \text { fare }_{t}+\beta_{1} \text { female }+\beta_{2} \text { age }+\varepsilon_{t} .
$$

We use a probit model to estimate the specification defined in (3.7.4). The results of this estimation are given in Table 3.7.1.

Table 3.7.1 Probit results for willingness-to-pay with data from the Titanic.

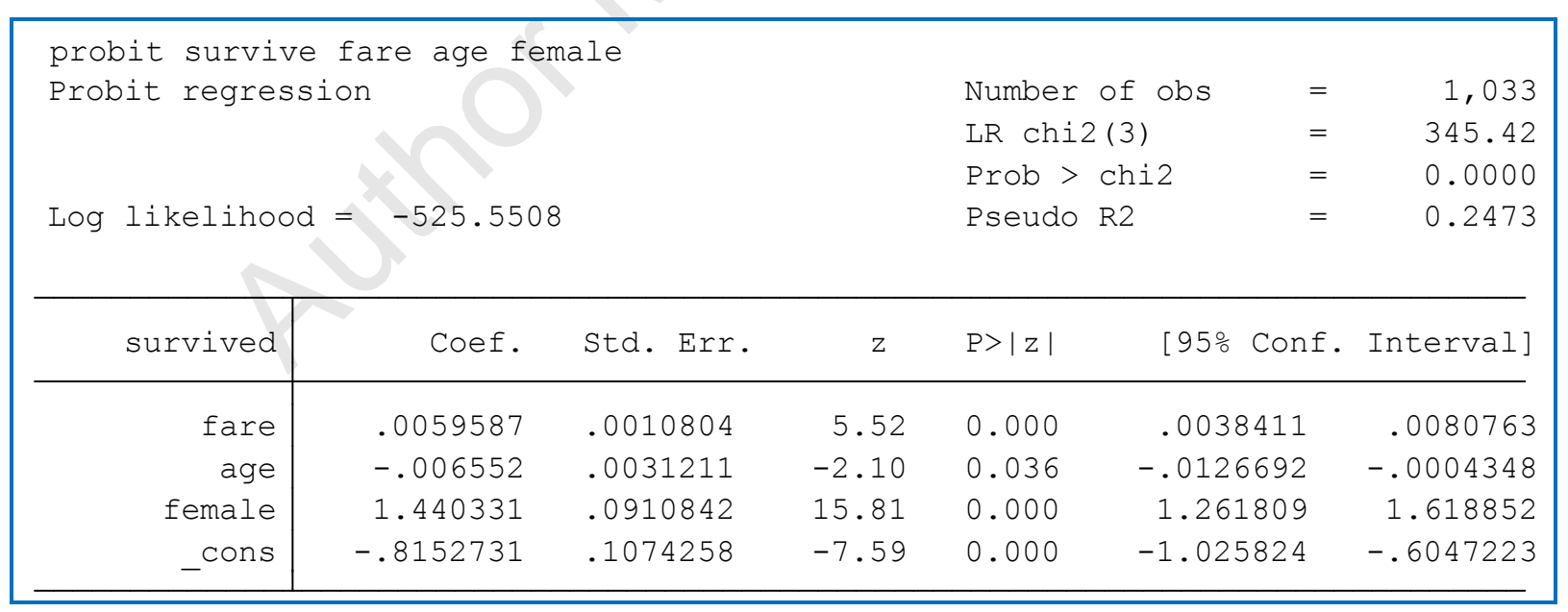

The Delta method CI for the $50 \%$ fare for the average age person ( $\mathrm{m}_{-}$age) and assuming 50\% gender split, results in the estimated CI on the $50 \%$ dose/willingness-to-pay defined by the post26

This article is protected by copyright. All rights reserved 
estimation results given in Table 3.7.2. The numerator is defined by $\hat{\rho}=-\left(\hat{\alpha}+.5 \hat{\beta}_{1}+\hat{\beta}_{2} m\right.$ age $)$ with a value of .290 and the denominator $\hat{\phi}=\hat{\gamma}$ with a value of .006 , and the expected willingnessto-pay is 48.7 pounds. From Table 3.7.2 we find that the approximate Delta $95 \%$ interval is from 32.818 to $64.538 .^{19}$

Table 3.7.2 The estimated willingness-to-pay and the corresponding Delta CI.

\begin{tabular}{|c|c|c|c|c|c|c|}
\hline \multirow{2}{*}{$\begin{array}{r}\text { rho: } \\
\text { phi: } \\
\text { theta: } \\
\text { survived }\end{array}$} & \multicolumn{6}{|c|}{ 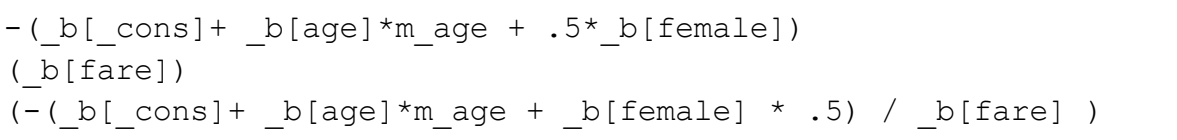 } \\
\hline & Coef. & Std. Err. & z & $P>|z|$ & [95\% Conf. & Interval] \\
\hline rho & .2900579 & .0583259 & 4.97 & 0.000 & .1757412 & .4043745 \\
\hline phi & .0059587 & .0010804 & 5.52 & 0.000 & .0038411 & .0080763 \\
\hline theta & 48.67789 & 8.092075 & 6.02 & 0.000 & 32.81771 & 64.53806 \\
\hline
\end{tabular}

To estimate the Fieller interval we use the margins command to predict the probability of survival, where the transformation of the linear combination of the parameters given by $\Phi^{-1}(\hat{\phi} \theta-\hat{\rho})=\Phi^{-1}\left(\hat{\gamma}\right.$ fare $+\left(\hat{\alpha}+.5 \hat{\beta}_{1}+\hat{\beta}_{2}\right.$ m_age $\left.)\right)$ provides the predicted probability of survival by differing values of the fare, (where $\Phi^{-1}$ indicates the inverse of the cumulative normal distribution defined by the normal() function in Stata). The 95\% Fieller CI bounds are estimated as 33.723 to 67.930 . 
Figure 3.7.1 The Fieller and Delta CIs for the 50\% level of the willingness to pay for survival on the Titanic

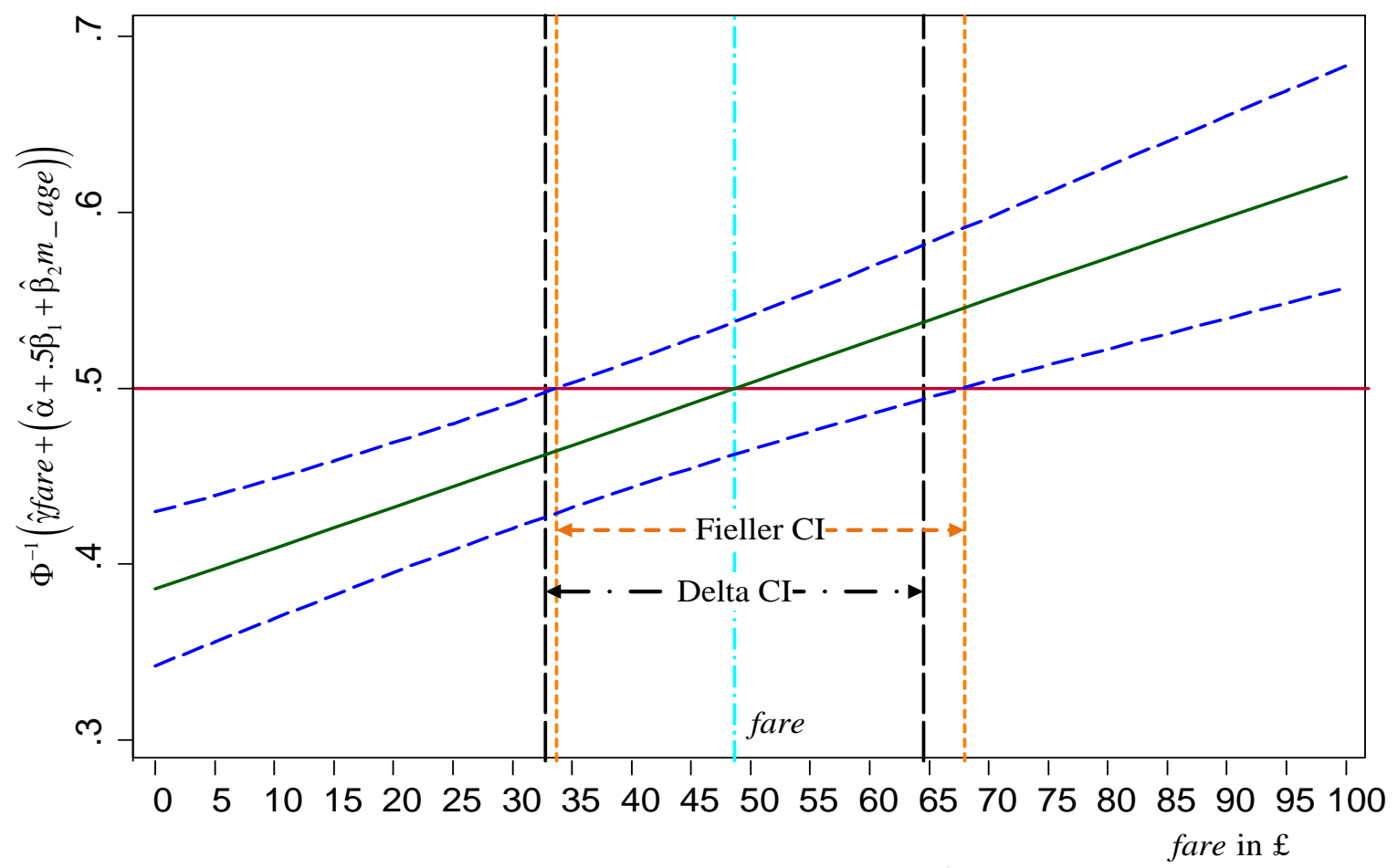

In this case the Delta and Fieller intervals coincide quite closely since the $t$-statistic on fare is 5.52 although the upper bound of the Fieller interval indicates slightly higher fares than the Delta. Note that in Figure 3.7.1 the reference line for the predicted probability of survival is put at .5. However, alternative levels could have been chosen or ranges of values i. e. between .4 and 6 instead.

\subsection{Indirect Least Squares as Two Stage Least Squares}

When a model is exactly identified with one endogenous regressor, the Two-Stage Least Squares (2SLS) and the instrumental variable (IV) estimates can be shown to be equivalent to the indirect least-squares (ILS) estimate of the structural parameter. Furthermore, when the ILS estimator is the ratio of two parameter estimates from equations that are estimated using seemingly unrelated regressions (SUR) the Delta CI is equivalent to the CI from the usual 2SLS estimation procedure. Hirschberg and Lye (2017) examine the Delta and Fieller CIs for the 2SLS/IV estimator of the coefficient of the endogenous regressor under these conditions.

Suppose the equation of interest is,

$$
Y_{i}=\beta_{0}+\beta_{1} X_{i}+\gamma_{1} W_{1 i}+\gamma_{2} W_{2 i}+\ldots \gamma_{K} W_{K i}+u_{i}
$$


where $Y$ is the dependent variable of interest, $X$ is an endogenous regressor vector, $W_{k}$ are the predetermined or exogenous regressors and $u$ is the error term. 2SLS/IV estimation requires $r$ instruments $Z_{1} \ldots Z_{r}(r \geq 1)$ that satisfy two conditions: (i) they are uncorrelated with $u$ and (ii) are correlated with $X$. When $r=1$ we say that (3.8.1) is exactly identified. In this case the reduced form equation of $X$ is,

$$
X_{i}=\pi_{10}+\pi_{11} Z_{1 i}+\psi_{11} W_{1 i}+\psi_{12} W_{2 i}+\ldots \psi_{1 K} W_{K i}+v_{1 i}
$$

and similarly, the reduced-form equation of $Y$ is defined by:

$$
Y_{i}=\pi_{20}+\pi_{21} Z_{1 i}+\psi_{21} W_{2 i}+\psi_{22} W_{2 i}+\ldots \psi_{2 K} W_{K i}+v_{2 i}
$$

The ILS estimator of $\beta_{1}$ in (3.8.1) is the ratio:

$$
\tilde{\theta}=\frac{\hat{\rho}}{\hat{\phi}}=\frac{\hat{\pi}_{21}}{\hat{\pi}_{11}}
$$

where $\hat{\pi}_{21}$ is the OLS estimator of $\pi_{21}$ in (3.8.3), $\hat{\pi}_{11}$ is the OLS estimator of $\pi_{11}$ in (3.8.2) and the covariance of these two parameter estimates is estimated via the application of the SUR technique.

For example, Acemoglu, etal (2001) examine the effect of institutions on economic performance. In this case the structural equation is,

$$
\log \left(y_{i}\right)=\mu+\beta R_{i}+\mathbf{X}_{i}^{\prime} \theta+u_{i}
$$

where $y_{i}$ is income per capita in country $i, R_{i}$ is the protection against expropriation measures and $\mathbf{X}_{i}$ is a vector of other covariates that includes dummy variables for the different continents on which the countries are located and the absolute value of their latitude.

Alternately we can consider the two reduced form equations. The protection against expropriation variable $R_{i}$ is treated as endogenous, with reduced form defined by:

$$
R_{i}=\pi_{10}+\pi_{11} \log \left(M_{i}\right)+\mathbf{X}_{i}^{\prime} \delta_{1}+v_{1 i}
$$

where $M_{i}$ is the settler mortality rate in 1,000 mean strength. The reduced form equation for $\log$ income per capita is

$$
\log \left(y_{i}\right)=\pi_{20}+\pi_{21} \log \left(M_{i}\right)+\mathbf{X}_{\mathbf{i}}^{\prime} \delta_{2}+v_{2 i}
$$

This model is just-identified with $\log \left(M_{i}\right)$ being excluded from (3.8.5). In this case the indirect least squares estimate of the structural parameter $\left(\tilde{\beta}_{I L S}\right)$ is defined as the ratio of the parameters: 


$$
\tilde{\beta}_{I L S}=\frac{\hat{\pi}_{21}}{\hat{\pi}_{11}}
$$

To demonstrate this equivalence of the 2SLS/IV and the ILS approaches, we provide the 2SLS/IV estimates for the model specified in (3.8.5) where $\log \left(M_{i}\right)$ is used as the instrument in Table 3.8.1. From this result, we note that the estimate for the structural parameter $(\beta)$ is given as 1.107 with a $95 \%$ CI of .242 to 1.972 and would result in the rejection of the null hypothesis that $\beta=0$ at the $5 \%$ level of significance.

Table 3.8.1 The Stata estimation results for the 2SLS application to the model where: logpgdp $95=\log \left(y_{i}\right), \operatorname{logem} 4=\log \left(M_{i}\right)$, avexpr $=R_{i}$, and africa, asia, lat_abst, other_cont $=\mathbf{X}_{i}$

\begin{tabular}{|c|c|c|c|c|c|c|c|}
\hline Instrumental & variables (2SL. & S) regress & & \multicolumn{2}{|c|}{$\begin{array}{l}\text { Number of obs } \\
\text { Wald chi2 (5) } \\
\text { Prob > chi2 } \\
\text { R-squared }\end{array}$} & $\begin{array}{l}= \\
= \\
= \\
= \\
=\end{array}$ & $\begin{array}{r}64 \\
37.78 \\
0.0000 \\
0.0108 \\
1.0296\end{array}$ \\
\hline logpgp95 & Coef. & Std. Err. & $\mathrm{z}$ & $\mathrm{P}>|z|$ & [95\% & Conf. & Interval] \\
\hline avexpr & 1.107077 & .4413078 & 2.51 & 0.012 & .24212 & 296 & 1.972024 \\
\hline africa & -.4372669 & .403835 & -1.08 & 0.279 & -1.228 & 3769 & .3542352 \\
\hline asia & -1.047085 & .499367 & $-2 \cdot 10$ & 0.036 & -2.0258 & 826 & -.0683438 \\
\hline lat_abst & -1.178178 & 1.671129 & -0.71 & 0.481 & -4.4535 & 3531 & 2.097174 \\
\hline other_cont & -.9904014 & .9500526 & -1.04 & 0.297 & -2.852 & 247 & .8716674 \\
\hline$-^{\text {cons }}$ & 1.440453 & 2.703206 & 0.53 & 0.594 & -3.857 & 732 & 6.738639 \\
\hline $\begin{array}{l}\text { Instrumented: } \\
\text { Instruments: }\end{array}$ & $\begin{array}{l}\text { avexpr } \\
\text { africa asia }\end{array}$ & lat_abst o & er_cont & logem4 & & & \\
\hline
\end{tabular}


Table 3.8.2 The Stata estimated parameters for the two reduced form equations via SUR.

\begin{tabular}{|c|c|c|c|c|c|c|}
\hline \multicolumn{7}{|c|}{ Seemingly unrelated regression } \\
\hline Equation & Obs & Parms & RMSE & "R-sq" & chi2 & $\mathrm{P}$ \\
\hline \multirow{2}{*}{$\begin{array}{l}\text { logpgp } 95 \\
\text { avexpr }\end{array}$} & 64 & $\begin{array}{l}5 \\
5\end{array}$ & $\begin{array}{l}.6677511 \\
1.194735\end{array}$ & $\begin{array}{l}0.5839 \\
3277\end{array}$ & 89.81 & 0.0000 \\
\hline & Coef. & Std. Err. & z & $\mathrm{P}>|\mathrm{z}|$ & [95 Conf. & Interval] \\
\hline \multicolumn{7}{|l|}{ logpgp 95} \\
\hline $\operatorname{logem} 4$ & -.3767581 & .0974069 & -3.87 & 0.000 & -.5676721 & -.185844 \\
\hline lat_abst & 1.046296 & .7400363 & 1.41 & 0.157 & -.4041483 & 2.496741 \\
\hline africa & -.7228463 & .2179443 & -3.32 & 0.001 & -1.150009 & -.2956833 \\
\hline asia & -.5245579 & .267608 & -1.96 & 0.050 & -1.04906 & -.0000559 \\
\hline other_cont & .1847984 & .4489049 & 0.41 & 0.681 & -.6950391 & 1.064636 \\
\hline _cons & 9.997378 & .5091475 & 19.64 & 0.000 & 8.999467 & 10.99529 \\
\hline \multicolumn{7}{|l|}{ avexpr } \\
\hline logem4 & -.3403178 & .1742797 & -1.95 & 0.051 & -.6818998 & .0012641 \\
\hline lat_abst & 2.009322 & 1.324067 & 1.52 & 0.129 & -.5858021 & 4.604447 \\
\hline africa & -.2579581 & .3899443 & -0.66 & 0.508 & -1.022235 & .5063187 \\
\hline asia & .4719882 & .4788021 & 0.99 & 0.324 & -.4664467 & 1.410423 \\
\hline other_cont & 1.061534 & .8031773 & 1.32 & 0.186 & -.5126647 & 2.635733 \\
\hline Cons & 7.729295 & .9109629 & 8.48 & 0.000 & 5.943841 & 9.51475 \\
\hline
\end{tabular}

In Table 3.8.2 we present the results of the SUR estimation for the two reduced form regressions. From (3.8.8) the ILS approach results in the estimate of the structural parameter as the ratio of the two parameters estimated for the logem4 regressor in the two equations. The Delta $95 \%$ CI for the ratio of these two parameter estimates is given in Table 3.8.3. From this table, we note that the ILS estimate of the structural parameter and its CI is identical to the 2SLS/IV estimate reported in Table 3.8.1. ${ }^{20}$

Table 3.8.3 The Stata results of the estimation of the ratio defined by $\tilde{\beta}_{I L S}=\frac{\hat{\pi}_{21}}{\hat{\pi}_{11}}$

\begin{tabular}{|c|c|c|c|c|c|c|}
\hline $\begin{array}{r}\text { rho: } \\
\text { phi: } \\
\text { theta: }\end{array}$ & $\begin{array}{r}\text { b [ logpgp } 95 \\
\text { b [avexpr: }] \\
\text { b [ logpgp } 95\end{array}$ & $\begin{array}{l}\text { Logem4 ] } \\
\text { gem4 ] } \\
\text { Logem4 ] / }\end{array}$ & avexpr & Logem 4 & & \\
\hline & Coef. & Std. Err. & z & $P>|z|$ & [95\% Conf. & Interval] \\
\hline rho & -.3767581 & .0974069 & -3.87 & 0.000 & -.5676721 & -.185844 \\
\hline phi & -.3403178 & .1742797 & -1.95 & 0.051 & -.6818998 & .0012641 \\
\hline theta & 1.107077 & .4413078 & 2.51 & 0.012 & .2421296 & 1.972024 \\
\hline
\end{tabular}


Figure 3.8.1 Fieller 95\% Confidence Interval for the structural parameter.

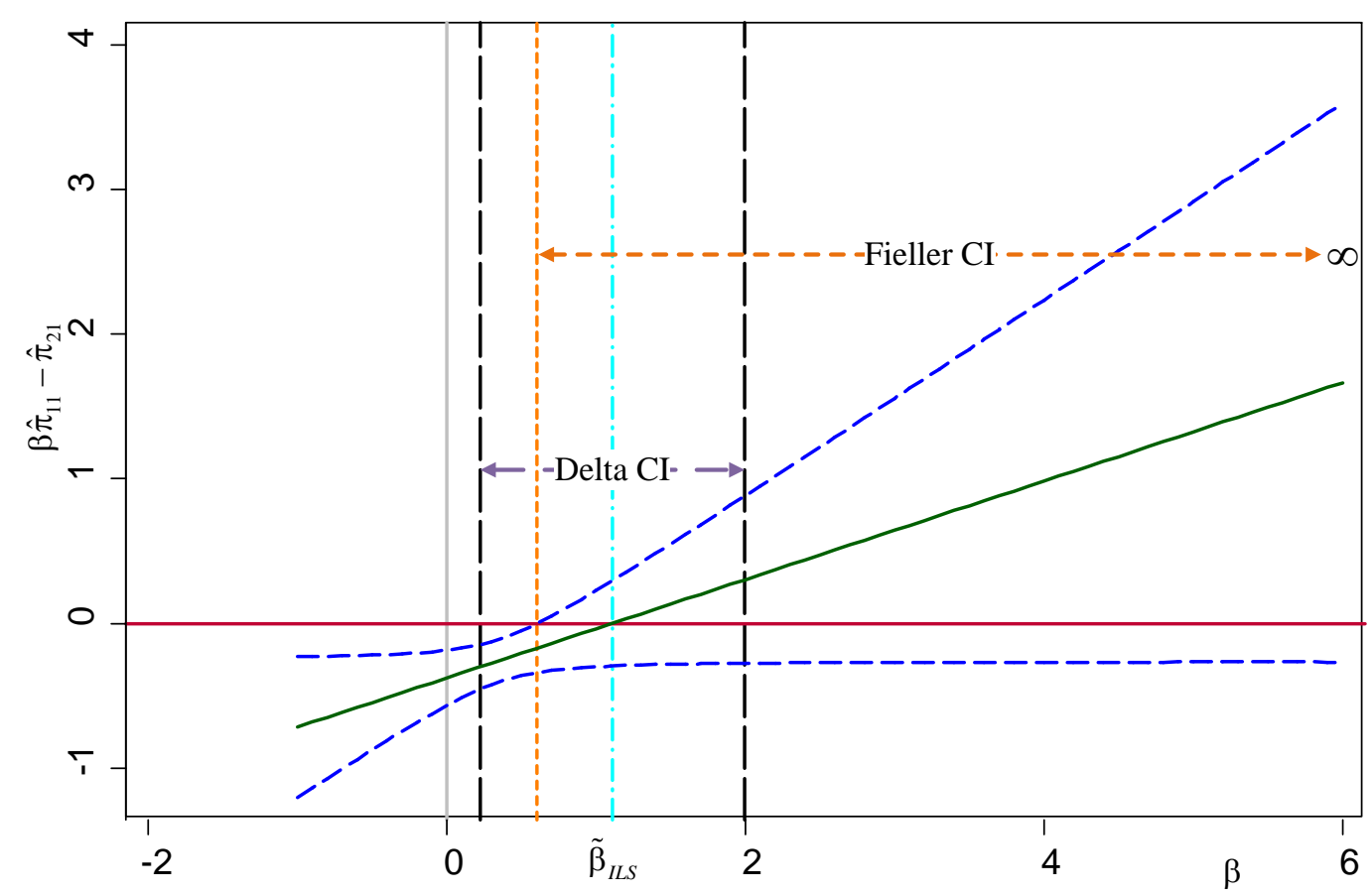

Following the derivation of the Fieller CI as discussed in Section 2.3, we find that there are two roots for the quadratic equation (2.3.4) as .609 to -200.843. We note that these two roots bear little similarity to the Delta bounds and the upper bound is estimated as below the lower bound. This is an example of the Fieller confidence interval resulting in the complement of a finite interval since in (2.3.4) $\left(b^{2}-4 a c\right)=.031>0$ and $a=-.006<0$. If we construct the line plot representation in the range of the Delta CI as shown in Figure 3.8.1, we find the appropriate interpretation of the Fieller CI is (.609 to $\infty$ ) or that the upper bound of the $95 \%$ Fieller CI is not defined. This would imply that we can reject the hypothesis that the parameter estimate is less than or equal to zero but there is no limit to the parameter estimate in the positive direction. A more thorough discussion of this result is available in Hirschberg and Lye (2017).

\subsection{Summary and Conclusions}

In this paper, we have presented a comparison between the Fieller and Delta methods for the approximation of the $\mathrm{CI}$ for cases where the quantity of interest is the ratio of a linear combination of parameter estimates. We present this method with emphasis on the application of the Stata statistical software routine. We also include programs in the R computer software in the supplemental materials along with a link to the datasets employed. ${ }^{21}$ The examples of the consideration of ratios has been shown in various econometric applications. 
One of the conclusions that can be drawn from these examples is that although the Fieller and the Delta CIs coincide in many cases, there are some situations where the use of the Fieller and Delta result in far different conclusions. This should not be surprising since both methods provide estimated intervals and not the true $\mathrm{CI}$ for a ratio of normally distributed random variables. Recent studies of the coverage of the CIs of the two methods has found that the Fieller CI conforms more closely to the true interval when Delta and the Fieller result in very different outcomes. Using the simple graphical method demonstrated in this paper, the Fieller can be used in any application which employs a ratio of parameter estimates. Our advice is to use both methods and when they differ, to use the Fieller interval that has been shown to provide the superior level of coverage. ${ }^{22}$

A general caveat that applies for both the Delta and the Fieller is the assumption that the parameter estimates are normally distributed or asymptotically normally distributed. As is usually the case, this assumption is dependent on many of the other assumptions implied by the correct specification of the functional forms and the error generating process. 


\section{End Notes}

1 Where $\alpha$ is the significance level of the test statistic.

${ }^{2}$ For most of the examples used here we assume a linear model such that $\mathbf{X}(\mathbf{B})=\mathbf{X B}$ and that errors are

identically and independently distributed which implies that $\Omega=\sigma^{2} \mathbf{I}$. However, this can be relaxed by using robust standard errors such as Huber-White standard errors, clustered standard errors or Newey-West standard errors.

3 This assumption may be relaxed to allow for the parameters to have an asymptotically normal distribution however the methods discussed here will then be subject to an additional degree of approximation.

4 These commands are similar to the ones used for the examples presented in Section 3. The complete programs and data used for each example application is included in the supplementary materials for this article along with the equivalent set of programs in $R$.

$5 \mathrm{~b}$ [_cons] indicates the estimate for the parameter $\beta_{1},{ }_{-}[\mathrm{x} 2]$ indicates the estimate for the parameter $\beta_{2}$ and $\left[\mathrm{b}[\mathrm{x} 3]\right.$ indicates the estimate for the parameter $\beta_{3}$.

${ }^{6}$ Note that we are not changing the values of $\mathrm{x}_{3}$ we are just using this as a temporary name - the margins command requires that the variable names used are same as one of the regressors in the equation.

7 The Delta CI for $\hat{\theta}$ listed by Stata is based on the standard normal instead of the $t$-distribution. In some cases, this is appropriate while in others it is not. We note when the Delta CI is based on the $t$-distribution.

8 This method for inverse tests is more fully developed for the turning points in higher order polynomials in Lye and Hirschberg (2012).

9 This Section follows the techniques presented in Hirschberg and Lye (2010b).

${ }^{10}$ Note that a similar result can be found for $w$.

11 These bounds are based on the $t$-distribution as opposed to the $z$-distribution used by the Stata $n l c o m$ routine as reported in Table 3.3.2.

12 Note that an assumption in the estimation of dynamic models of this form with OLS is that the errors are not subject to autocorrelation.

13 Because the non-linear estimation parameters are asymptotically normally distributed we employ the $z$ statistic for both the Fieller and Delta intervals.

${ }^{14}$ See Hirschberg and Lye (2010c) for more details on the nature of the relationship between the CIs for both methods. In general, when the denominator is significantly different from zero and the correlation between the denominator and the numerator is the same sign as the ratio the Delta and the Fieller will tend to coincide.

15 See https://www.philadelphiafed.org/research-and-data/real-time-center/greenbook-data/ .

16 This section is based on materials from Hirschberg and Lye (2010a).

17 Note that $G^{-1}(.5)=0$ for both the logit and probit models and $G^{-1}(.5)=.5$ for the linear probability model.

18 Since the passengers did not know what the possibility of the disaster was prior to purchasing the ticket this is not a traditional willingness-to-pay problem but more akin to a 50\% dose model.

19 In this case the appropriate distribution for the CI is the standard normal $z$ because these parameters are the result of a maximum likelihood estimation procedure and are thus asymptotically normally distributed.

${ }^{20}$ Note that since the regressors in these equations are the same the estimated parameters from the SUR are identical to the OLS results if they were estimated separately. However, the SUR approach provides an estimate of the covariance between the parameter estimates of the two equations that is required for the correct estimation of the Delta and Fieller CIs. In this case we would use the standard normal distribution since the parameter estimates are asymptotically normally distributed.

21 The programs are listed in the working paper that can be found in the working paper "Confidence intervals for ratios: Econometric Examples in Stata and $\mathrm{R}$ that can be found at: https://fbe.unimelb.edu.au/_data/assets/pdf file/0005/2704649/2037 Joe-Hirschberg_Fieller-Examples-WPversion.pdf The data can be found at: https://data.mendeley.com/datasets/6t7mchv462/1.

${ }^{22}$ The analysis of the difference between these two methods is discussed in Hirschberg and Lye 2010c,2017. 


\section{References}

Acemoglu, D., S. Johnson and A. Robinson (2001), "The Colonial Origins of Comparative Development: An Empirical Investigation”, American Economic Review, 91, 1369-1401.

Bernard J-T, N. Idoudi, L. Khalaf and C. Yélou (2007), "Finite sample inference methods for dynamic energy demand models", Journal of Applied Econometrics 22,1211-1226

Buonaccorsi, J. P., (1979), "On Fieller's Theorem and the General Linear Model”, The American Statistician, 33, 162.

Cassella, G., and Berger, R. L. (2002), Statistical Inference (2nd ed.), Pacific Grove, CA.: Duxbury.

Coibon, O. and Y. Gorodnichenko (2011), "Monetary Policy, Trend Inflation, and the Great Moderation: An Alternative Interpretation", American Economic Review, 101, 341-370.

Fieller, E. C. (1932), “The Distribution of the Index in a Normal Bivariate Population”, Biometrika, 24, 428-440.

Fieller, E. C. (1944), "A Fundamental Formula in the Statistics of Biological Assay, and Some Applications”, Quarterly Journal of Pharmacy and Pharmacology, 17, 117-123.

Fieller, E. C. (1954), "Some Problems in Interval Estimation”, Journal of the Royal Statistical Society, Series B, 16, 174-185.

Finney, D.J. (1978) Statistica Methods in Biological Assay, Charles Griffin and Company, London.

Frey, B. S., D. A. Savage, and B. Torgler (2010), "Interaction of natural survival instincts and internalized social norms exploring the Titanic and Lusitania disasters", Proceedings of the National Academy of Science, 107, 4862-4865.

Gruen, D., A. Pagan and C. Thompson (1999), "The Phillips curve in Australia", Journal of Monetary Economics, 44, 223-258.

Harbaugh, W., A. Levinson and D. Wilson (2002), "Re-examining the Empirical Evidence for an Environmental Kuznets Curve", Review of Economics and Statistics, 84, 541-551.

Hirschberg J. and J. Lye (2010a), "Two geometric representations of confidence intervals for ratios of linear combinations of regression parameters: An application of the NAIRU", Economics Letters, 108, 73-76.

Hirschberg J. and J. Lye (2010b), "A reinterpretation of interactions in regressions", Applied Economics Letters, 17, 427-430.

Hirschberg J. and J. Lye (2010c), "A Geometric Comparison of the Delta and Fieller Confidence Intervals" (2010), The American Statistician, 64, 234-241.

Hirschberg J. and J. Lye (2010d), "The Indirect Impacts of Smoking Bans in Gaming Venues", 2010, chapter 11, Current Issues in Health Economics, edited by Daniel Slottje and Rusty Tchernis, Contributions to Economic Analysis, 290, Emerald Press, 243-259.

Hirschberg J. and J. Lye (2017), "Inverting the indirect-The ellipse and the boomerang: Visualizing the confidence intervals of the structural coefficient from two-stage least squares", Journal of Econometrics, 199, 173-183.

Hole, A. R. (2007), "A comparison of approaches to estimating confidence intervals for willingness to pay measures", Health Economics, 16, 827-840. 
Kuznets, S. (1955), "Economic Growth and Income Inequality", American Economic Review, 45, $1-28$.

Lye, J. and J. Hirschberg (2012), "Inverse test confidence intervals for turning-points: A demonstration with higher order polynomials", Advances in Econometrics, ed. D. Terrell and D. Millimet, 30, 59-95.

Modigliani, F. and L. Papademos (1975), "Targets for Monetary Policy in the Coming Year", Brookings Papers on Economic Activity, The Brookings Institution. 1975 (1): 141-165.

Phillips, A. W. (1958), “The Relationship between Unemployment and the Rate of Change of Money Wages in the United Kingdom 1861-1957", Economica, 25 (100): 283-299.

Rao, C. R. (1973), Linear Inference and its Applications, 2nd edition, New York: John Wiley and Sons.

Scheffé, H. (1970), "Multiple testing versus multiple estimation. Improper confidence sets. Estimation of directions and ratios", The Annals of Mathematical Statistics, 41, 1-29.

Stock, J. H. and M. W. Watson, (2003), Introduction to Econometrics, Addison Wesley, USA.

Taylor, J. B. (1993), "Discretion versus Policy Rules in Practice". Carnegie-Rochester Conference Series on Public Policy. 39: 195-214

Zerbe, G., (1982), “On Multivariate Confidence Regions and Simultaneous Confidence Limits for Ratios”, Communications in Statistics Theory and Methods, 11, 2401-2425. 


\section{University Library}

\section{- M M N E R VA A gateway to Melbourne's research publications}

Minerva Access is the Institutional Repository of The University of Melbourne

Author/s:

Lye, J;Hirschberg, J

Title:

Ratios of Parameters: Some Econometric Examples

Date:

2018-12-01

Citation:

Lye, J. \& Hirschberg, J. (2018). Ratios of Parameters: Some Econometric

Examples. AUSTRALIAN ECONOMIC REVIEW, 51 (4), pp.578-602. https:// doi.org/10.1111/1467-8462.12300.

Persistent Link:

http://hdl.handle.net/11343/284806 